\title{
查尔酮衍生物的合成及其抑菌活性和分子对接研究
}

\author{
肖婷婷程玮钱伟烽张婷婷 \\ 陆童高扬唐孝荣* \\ (西华大学理学院 成都 610039)
}

\begin{abstract}
摘要 设计合成了 29 个查尔酮衍生物, 并用 IR、 ${ }^{1} \mathrm{H} \mathrm{NMR} 、{ }^{13} \mathrm{C} N \mathrm{NMR}$ 和 HRMS 对其结构进行了表征. 测定了所有合成 化合物对 5 种植物病原真菌即水稻纹枯病原真菌、小麦赤霉病原真菌、玉米小玟病原真菌、油菜菌核病原真菌和番茄 灰霉病原真菌的抑菌活性. 初步的结果显示, 大多数化合物都有显著的抑菌活性，其中, (E)- $N$-(4-(3-(5-澳噻吩-2-基)丙 烯酰基)苯基)烟酰胺 $(\mathbf{4 m})\left(\mathrm{EC}_{50}=0.057 \mathrm{mg} / \mathrm{L}\right.$ )和 $(E)$-2-羊基- $N$-(4-(3-(吡啶-3-基)丙烯酰基)苯基)乙酰胺(6i) $\left(\mathrm{EC}_{50}=0.054\right.$ $\mathrm{mg} / \mathrm{L})$ 对油菜菌核表现出最强的抑制活性, 活性优于市售杀菌剂氟吡菌酰胺 $\left(\mathrm{EC}_{50}=0.244 \mathrm{mg} / \mathrm{L}\right)$. 与此同时, 还测定了 化合物 $4 \mathrm{~m}$ 和 $6 \mathrm{i}$ 对琥珀酸脱氢酶 $(\mathrm{SDH})$ 的抑制活性. 结果显示, 它们都比氟吡菌酰胺有更好的抑制活性. 分子对接研究 表明, 化合物 $4 \mathbf{m}$ 和 $6 \mathbf{i}$ 都与 SDH 结合得很好, 结合能分别为 -31.0 和 $-31.4 \mathrm{~kJ} / \mathrm{mol}$. 而且, 化合物 $4 \mathbf{m}$ 和 $\mathbf{6} \mathbf{i}$ 分别与 SDH 的 B/Trp-230 残基形成了一个氢键.
\end{abstract}

关键词 抑菌活性; 查尔酮衍生物; 分子对接; 琥珀酸脱氢酶抑制剂

\section{Synthesis of Chalcone Derivatives and Studies on Their Inhibitory Activity and Molecular Docking}

\author{
Xiao, Tingting \\ Cheng, Wei \\ Qian, Weifeng \\ Zhang, Tingting \\ Lu, Tong \\ Gao, Yang Tang, Xiaorong* \\ (School of Science, Xihua University, Chengdu 610039)
}

\begin{abstract}
Twenty nine chalcone derivatives were designed, synthesized and characterized by IR, ${ }^{1} \mathrm{H} N \mathrm{NM},{ }^{13} \mathrm{C}$ NMR and HRMS. The antifungal activities of all the synthesized compounds were determined against five plant pathogenic fungi namely Rhizoctonia solani, Fusarum graminearum, Helminthosporium maydis, Sclerotinia sclerotiorum and Botrytis cinerea. Preliminary results indicated that most of them revealed significant antifungal activity. Among them, $(E)-N-(4-(3-(5-b r o m o t h i o p h e n-2-$ yl)acryloyl)phenyl)nicotinamide $(\mathbf{4 m})\left(\mathrm{EC}_{50}=0.057 \mathrm{mg} / \mathrm{L}\right)$ and $(E)$-2-hydroxy- $N$-(4-(3-(pyridin-3-yl)acryloyl)phenyl)acetamide $(6 \mathbf{6 i})\left(\mathrm{EC}_{50}=0.054 \mathrm{mg} / \mathrm{L}\right)$ showed the strongest activities against $S$. sclerotiorum and possessed better antifungal activities than the commercial fungicide of fluopyram $\left(\mathrm{EC}_{50}=0.244 \mathrm{mg} / \mathrm{L}\right)$. Meanwhile, the inhibitory activities of compounds $\mathbf{4 m}$ and $6 \mathbf{i}$ were tested against succinate dehydrogenase (SDH). The results displayed that they had also better activities than fluopyram. Molecular docking studies demonstrated that compounds $\mathbf{4 m}$ and $\mathbf{6 i}$ bound well to SDH and their binding energies were -31.0 and $-31.4 \mathrm{~kJ} / \mathrm{mol}$, respectively. Moreover, compounds $4 \mathrm{~m}$ and $\mathbf{6 i}$ formed hydrogen bonds with residue B/Trp-230 of SDH, respectively.
\end{abstract}

Keywords antifungal activity; chalcone derivatives; molecular docking; succinate dehydrogenase inhibitor

杀菌剂在控制植物病原体的生长和繁殖中发挥着 重要的作用. 琥珀酸脱氢酶(SDH)抑制剂是一类重要的 杀菌剂，现已广泛地用于控制包括水稻纹枯、油菜菌核
和番茄灰霉在内的植物病原真菌 ${ }^{[1 \sim 3]}$. 琥珀酸脱氢酶抑 制剂通过破坏线粒体的三羧酸循环和呼吸链而起作 用 ${ }^{[4]}$. 而且，独特的作用方式使得它们与其它市售杀菌

\footnotetext{
* Corresponding author. E-mail: tangxr112@126.com

Received November 26, 2019; revised January 11, 2020; published online February 23, 2020.

Project supported by the Technical Innovation Programs of Chengdu Municipal Bureau of Science and Technology (No. 2018-YF05-00970-SN), the Innovation Fund of Postgraduate of Xihua University (No. YCJJ2019025) and the Undergraduate Innovation and Entrepreneurship Training Programs of Xihua University (No. 201810623009).

成都市科学技术局技术创新(No. 2018-YF05-00970-SN)、西华大学研究生创新基金(No. YCJJ2019025)和西华大学大学生创新创业训练计划(No. 201810623009)资助项目。
} 
剂没有交互抗性 ${ }^{[4]}$. 因此, 琥珀酸脱氢酶抑制剂在全世 界都得到了广泛的研究 ${ }^{[5-8]}$. 自 1966 年第一个琥珀酸脱 氢酶抑制剂萎锈灵使用以来, 已成功地开发和使用了 18 个琥珀酸脱氢酶抑制剂. 与此同时, 琥珀酸脱氢酶抑 制剂的研究和创新一直是杀菌剂领域的热点和前沿, 不 断有新的品种被报道 ${ }^{[9 \sim 12]}$. 这些琥珀酸脱氢酶抑制剂在 分子结构上具有以下共同特征: 酰胺骨架、杂环或氟原 子(图 1).

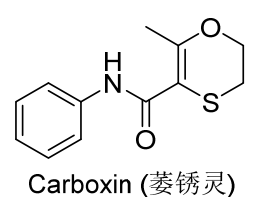<smiles>Cc1nc(C(F)(F)F)c(C(=O)Nc2c(Br)cc(OC(F)(F)F)cc2Br)s1</smiles>

Thifluzamide (噻呋酰胺)<smiles>O=C(NCCc1ncc(C(F)(F)F)cc1Cl)c1ccccc1C(F)(F)F</smiles>

Fluopyram (氟吡菌酰胺)<smiles>Cc1occc1C(=O)Nc1ccccc1</smiles>

Fenfuram (甲呋酰胺)<smiles>O=C(Nc1ccccc1-c1ccc(Cl)cc1)c1cccnc1Cl</smiles>

$\mathrm{Cl}$ Boscalid (啶酰菌胺)<smiles>CCOc1ccc(F)c(F)c1</smiles>

Fluxapyroxad (氟唑菌酰胺)
图 1 一些具有代表性的琥珀酸脱氢酶抑制剂

Figure 1 Representatives of succinate dehydrogenase inhibitors

查尔酮是一类存在于食用及药用植物中的结构多 样的天然产物, 它们可以通过化学合成的方法得到. 它 们由于简单而特殊的结构, 在化学合成中广泛地用作合 成子. 与此同时, 查尔酮衍生物由于具有包括抑菌(图

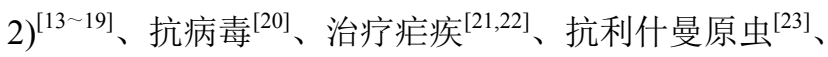
抗氧化 ${ }^{[24,25]}$ 、治疗糖尿病 ${ }^{[25,26]}$ 、抗癌 ${ }^{[27,28]}$ 、消炎 ${ }^{[29]}$ 和抗 结核 ${ }^{[30]}$ 等在内的多种生物活性, 得到了人们的广泛关 注. 到目前为止, 查尔酮的结构修饰及其生物活性的研 究工作仍在不断的进行之中.

在本研究中, 将杂环及酰胺骨架引入到查尔酮分子 结构中, 设计并合成了 29 个查尔酮衍生物, 其目的是发 现结构简单、性能优异的新型琥珀酸脱氢酶抑制剂(图 3). 与此同时, 对合成化合物的抑菌活性进行了实验室 测定, 并对其进行了分子对接研究, 以确定其抑菌活性 的关键结构所在.

\section{1 结果与讨论}

\section{1 化学合成}

设计的目标化合物的合成及其化学结构如 Scheme

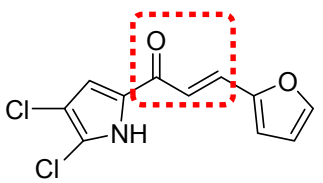

MIC $(\mathrm{mg} / \mathrm{L})=3.125$ against Escherichia coli

MIC $(\mathrm{mg} / \mathrm{L})=1.560$ against Staphylococcus aureus

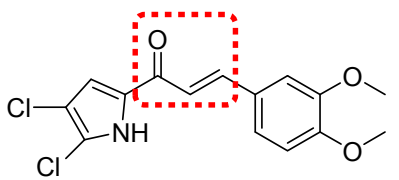

MIC $(\mathrm{mg} / \mathrm{L})=3.125$ against Staphylococcus aureus

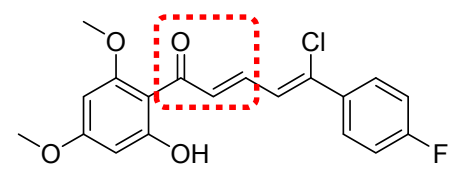

MIC $(\mathrm{mg} / \mathrm{mL})=25$ against Bacillius subtilis MIC $(\mathrm{mg} / \mathrm{mL})=25$ against Aspergillus niger

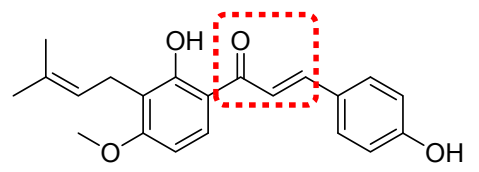

MIC $(\mathrm{mg} / \mathrm{L})=2.0$ against Bacillius subtilis, Micrococcus luteus

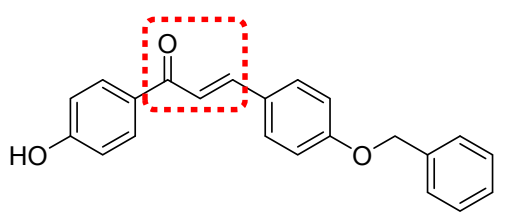

MIC $(\mathrm{mg} / \mathrm{L})=3.960$ against Escherichia coli

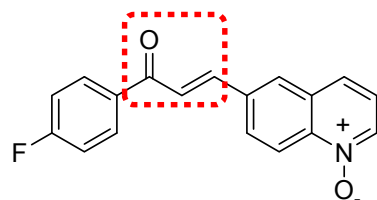

MIC $(\mathrm{mg} / \mathrm{L})=1.90$ against Paracoccidioides brasiliensis

图 2 一些具有抑菌活性的查尔酮衍生物

Figure 2 Some chalcone derivatives with antibacterial or antifungal activity

1 所示. 中间体 1、2 和 3 的合成采用文献报道的方法进 行, 且表征数据与文献一致 ${ }^{[31 ~ 35]}$. 中间体 $\mathbf{1} 、 2$ 和 3 的合 成是通过相应酰氯的氨解反应完成的. 化合物 $4 \mathrm{a} \sim \mathbf{4 m}$ 的合成也是通过相应酰氯的氨解反应完成的. 化合物 $5 \mathrm{a} \sim 5 \mathrm{~g}$ 的合成方法如下: 在进行克莱森-施密特缩合反 应的同时，溶剂中的乙醇选择性地和丙烯酰胺基中的共 轭 $\mathrm{C}=\mathrm{C} 、 \mathrm{C}=\mathrm{O}$ 双键发生迈克尔加成反应, 这是因为丙 烯酰胺基中共轭的 $\mathrm{C}=\mathrm{C} 、 \mathrm{C}=\mathrm{O}$ 双键的空间位阻比另一 个连接在苯环上的共轭的 $\mathrm{C}=\mathrm{C} 、 \mathrm{C}=\mathrm{O}$ 双键的要小. 化 合物 $6 \mathbf{a} \sim 6 \mathbf{i}$ 也是通过两个同时进行的反应(克莱森-施 密特缩合反应和乙酰氧基的水解反应)合成. 用 $I R 、{ }^{1} \mathrm{H}$ 


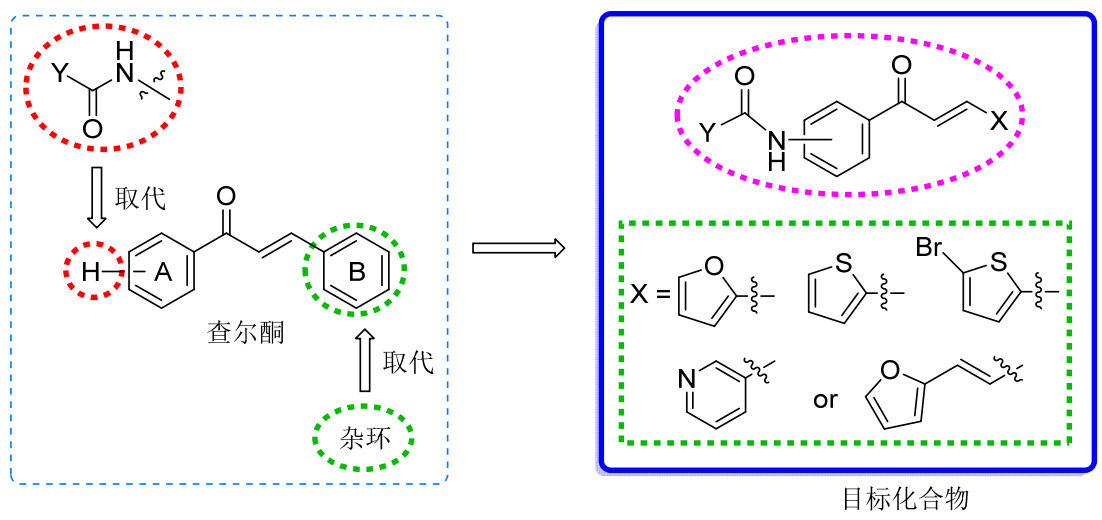

图 3 目标化合物的设计

Figure 3 Design of target compounds

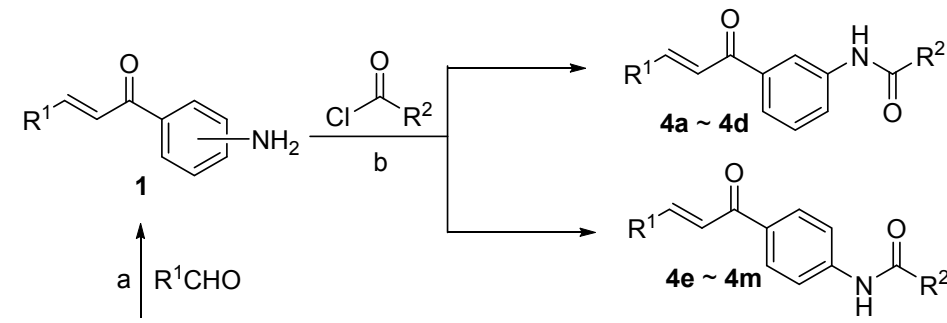<smiles>[R10]C(C)[C@H](CC)[C@H](CC)OC(C)=O</smiles>

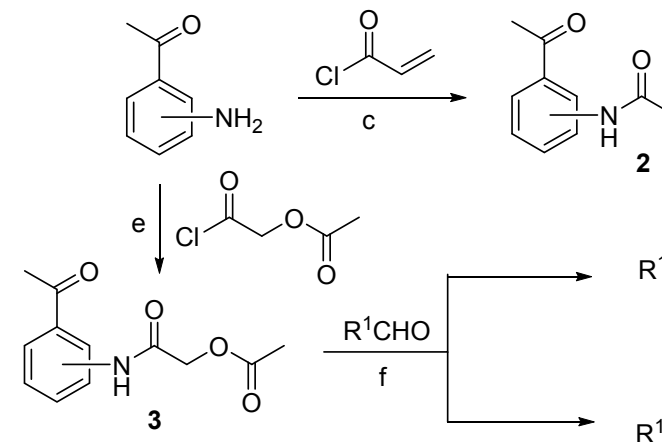
$\mathrm{R}^{2}$

2<smiles>[R]C=CC(=O)c1cccc(NC(=O)CO)c1</smiles><smiles>[R]C=CC(=O)c1ccc(NC(=O)CCOCC)cc1</smiles>
$5 d \sim 5 g$<smiles>[R]C=CC(=O)c1ccc(NC(=O)CO)cc1</smiles>

4a: $\left.\mathrm{R}^{1}={ }_{\mathrm{O}}\right\}$

4b: $R^{1}=\left\langle{ }_{S} \xi-R^{2}=\mathrm{H}_{2} \mathrm{C}=\mathrm{CH}-\right.$

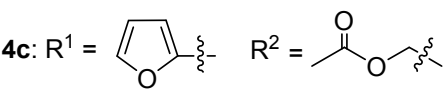

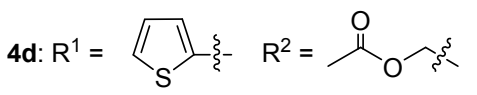

4e: $\mathrm{R}^{1}=4 \mathrm{O}_{\mathrm{O}} \mathrm{R}^{2}=\mathrm{H}_{2} \mathrm{C}=\mathrm{CH}$

4f: $R^{1}=\left\langle{ }_{S} \| \xi-R^{2}=\mathrm{H}_{2} \mathrm{C}=\mathrm{CH}-\right.$

4g: $\mathrm{R}^{1}=\mathrm{R}_{\mathrm{O}}^{2}=\mathrm{H}_{2} \mathrm{C}=\mathrm{CH}-$

4h: $\mathrm{R}^{1}=$

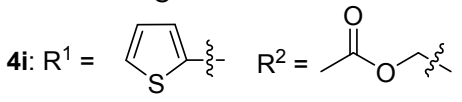

$4 \mathrm{j}: \mathrm{R}^{1}=$ N- $\mathrm{R}^{2}=\mathrm{N}_{\mathrm{O}}$

4k: $R^{1}=\left\langle R_{0}^{1} R^{2}=\right.$

4I: $R^{1}=\left\langle R_{S} \xi R^{2}=\right.$

$4 \mathrm{~m}: \mathrm{R}^{1}=\mathrm{Br} \mathrm{S}_{\mathrm{S}}$

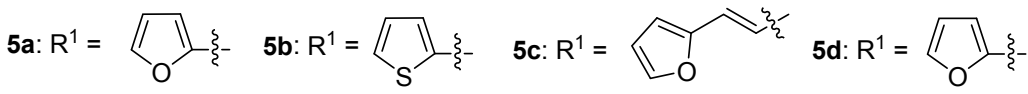

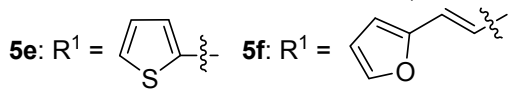

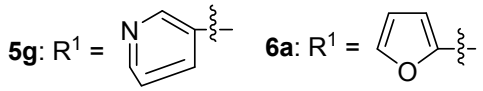

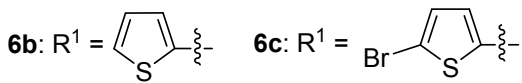

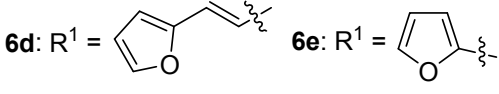

6f: $R^{1}=\left\langle\left\|_{S}\right\|_{-} \quad 6 g: R^{1}={ }_{B r}\right.$

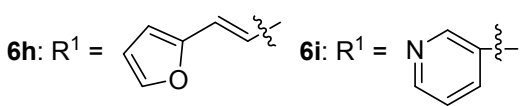

Reagents and conditions: (a) EtOH, $10 \% \mathrm{NaOH}, 0 \sim 5{ }^{\circ} \mathrm{C}, 3 \sim 5 \mathrm{~h}$; (b) $\mathrm{CH}_{2} \mathrm{Cl}_{2}, \mathrm{Et}_{3} \mathrm{~N}, 0 \sim 5{ }^{\circ} \mathrm{C}, 2 \sim 4 \mathrm{~h}$; (c) $\mathrm{CH}_{2} \mathrm{Cl}_{2}, \mathrm{Et} \mathrm{t}_{3} \mathrm{~N}, 0 \sim 5{ }^{\circ} \mathrm{C}, 2 \sim 4 \mathrm{~h}$; (d) $\mathrm{EtOH}, 10 \%$ $\mathrm{NaOH}, 45 \sim 50{ }^{\circ} \mathrm{C}, 3 \sim 5 \mathrm{~h}$; (e) $\mathrm{CH}_{2} \mathrm{Cl}_{2}, \mathrm{Et}_{3} \mathrm{~N}, 0 \sim 5{ }^{\circ} \mathrm{C}, 3 \sim 5 \mathrm{~h}$; (f) EtOH, $10 \% \mathrm{NaOH}, 45 \sim 50{ }^{\circ} \mathrm{C}, 4 \sim 6 \mathrm{~h}$

图式 1 目标化合物的合成

Scheme 1 Synthesis of target compounds 
NMR、 ${ }^{13} \mathrm{C}$ NMR 和 HRMS 对所合成的化合物的结构进 行了表征. 结果表明, 它们的波谱数据与所假定的结构 相符合.

\section{2 抑菌活性}

采用高效杀菌剂氟吡菌酰胺为阳性对照, 以水稻纹 枯病原真菌 $(R$. solani)、小麦赤霉病原真菌 $(F$. graminearum)、玉米小斑病原真菌(H. maydis)、油菜菌核病原真 菌(S. sclerotiorum) 和番茄灰霉病原真菌 (B. cinerea) 为靶 标, 对所有合成化合物的抑菌活性进行了生物活性测 试, 结果如表 1 所示. 从表中可知, 大多数化合物都有 显著的抑菌活性. 其中, 化合物 $4 \mathrm{a} 、 4 \mathrm{~b} 、 4 \mathrm{~d} \sim 4 \mathrm{f} 、 4 \mathrm{~m}$ 、 $5 a \sim 5 d 、 5 f 、 5 g 、 6 c$ 和 $6 i$ 具有优异的抑菌活性. 特别是 化合物 $4 \mathrm{a} 、 4 \mathrm{~b} 、 4 \mathrm{~m} 、 5 \mathrm{~g}$ 和 $6 \mathbf{i}$ 在 $20 \mathrm{mg} / \mathrm{L}$ 时对油菜菌核 病原真菌的抑菌率达到了 $100 \%$.

如表 1 所示，合成的所有化合物对上述 5 种真菌的 抑菌活性大小顺序为: 油菜菌核病原真菌 $>$ 玉米小斑病
原真菌 $>$ 水稻纹枯病原真菌 $>$ 小麦赤霉病原真菌 $>$ 番 茄灰霉病原真菌. 如果将化合物 4l、6b、6f 的抑菌活性 分别与化合物 $4 \mathrm{~m} 、 6 \mathrm{c} 、 6 \mathrm{~g}$ 的抑菌活性进行比较, 可发 现当噻吩环 5 位上的氢原子被溴原子取代后，相应化合 物的抑菌活性有所增强, 其原因还有待于进一步研究. 为了进一步研究目标化合物的抑菌活性, 测定了对油菜 菌核病原真菌具有优异抑制效果的化合物的半数有效 浓度 $\left(\mathrm{EC}_{50}\right)$ 值，结果如表 2 所示. 化合物 $4 \mathbf{a} 、 \mathbf{4 b} 、 \mathbf{4 d} \sim$

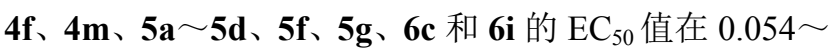
$1.420 \mathrm{mg} / \mathrm{L}$. 其中, 化合物 $\mathbf{4 m}$ 和 $\mathbf{6 i}$ 的 $\mathrm{EC}_{50}$ 值分别达到 了 0.057 和 $0.054 \mathrm{mg} / \mathrm{L}$, 而氟吡定的 $\mathrm{EC}_{50}$ 值为 0.244 $\mathrm{mg} / \mathrm{L}$, 这表示化合物 $\mathbf{4 m}$ 和 $6 \mathbf{i}$ 比氟吡菌酰胺具有更好的 抑菌活性. 同样, 化合物 $\mathbf{4 a} 、 \mathbf{4 b}$ 和 $\mathbf{5 g}$ 的 $\mathrm{EC}_{50}$ 值分别为 $0.305 、 0.282$ 和 $0.294 \mathrm{mg} / \mathrm{L}$, 表明它们的抑菌活性接近 氟吡菌酰胺. 此外, 如果将 $4 a 、 4 b 、 5 c$ 对油菜菌核的抑 制活性分别同化合物 $4 \mathrm{e} 、 4 \mathrm{f} 、 5 \mathrm{f}$ 的抑制活性进行比较, 可

表 1 目标化合物在 $20 \mathrm{mg} / \mathrm{L}$ 的抑菌活性

Table 1 Antifungal activity of target compounds at $20 \mathrm{mg} / \mathrm{L}$

\begin{tabular}{|c|c|c|c|c|c|}
\hline \multirow{2}{*}{ Compd. } & \multicolumn{5}{|c|}{ Inhibition rate ${ }^{a} / \%$} \\
\hline & R. solani & F. graminearum & H. maydis & S. sclerotiorum & B. cinerea \\
\hline $4 a$ & 56.78 & 47.12 & 86.81 & 100.00 & 40.56 \\
\hline $4 b$ & 70.04 & 61.46 & 88.56 & 100.00 & 56.60 \\
\hline $4 c$ & 55.96 & 49.27 & 56.90 & 65.81 & 38.35 \\
\hline 4d & 69.40 & 58.93 & 78.06 & 92.14 & 43.52 \\
\hline $4 e$ & 70.62 & 53.94 & 83.57 & 97.38 & 44.61 \\
\hline $4 f$ & 62.76 & 56.78 & 82.34 & 96.53 & 42.89 \\
\hline $4 \mathrm{~g}$ & 68.21 & 56.32 & 70.05 & 74.89 & 45.68 \\
\hline $4 h$ & 42.09 & 36.67 & 45.28 & 51.39 & 29.57 \\
\hline $4 i$ & 66.73 & 56.89 & 69.03 & 73.81 & 33.13 \\
\hline $4 j$ & 57.78 & 51.26 & 58.60 & 71.85 & 42.21 \\
\hline $4 k$ & 58.31 & 45.61 & 61.06 & 78.08 & 32.14 \\
\hline 41 & 41.23 & 38.05 & 52.71 & 55.05 & 28.33 \\
\hline $4 m$ & 77.76 & 68.30 & 96.09 & 100.00 & 58.42 \\
\hline $5 \mathbf{a}$ & 69.16 & 60.86 & 78.96 & 90.36 & 43.17 \\
\hline $5 \mathbf{b}$ & 68.75 & 61.95 & 75.67 & 96.75 & 41.29 \\
\hline $5 c$ & 67.63 & 59.38 & 83.25 & 97.21 & 40.63 \\
\hline $5 d$ & 65.42 & 52.03 & 73.20 & 96.26 & 38.74 \\
\hline $5 e$ & 55.74 & 43.15 & 58.02 & 69.60 & 41.28 \\
\hline $5 f$ & 70.46 & 60.20 & 76.28 & 92.07 & 40.61 \\
\hline $5 \mathrm{~g}$ & 69.38 & 60.47 & 87.21 & 100.00 & 52.06 \\
\hline $6 a$ & 52.19 & 36.81 & 58.49 & 65.85 & 26.72 \\
\hline $6 \mathbf{b}$ & 48.86 & 38.74 & 52.13 & 56.02 & 25.47 \\
\hline $6 c$ & 61.76 & 48.95 & 73.32 & 90.19 & 38.45 \\
\hline $6 d$ & 42.01 & 34.58 & 55.26 & 60.17 & 26.79 \\
\hline $6 e$ & 46.52 & 41.30 & 51.86 & 57.41 & 25.23 \\
\hline $6 f$ & 53.69 & 47.33 & 59.02 & 63.29 & 28.36 \\
\hline $6 \mathrm{~g}$ & 64.17 & 59.80 & 68.93 & 72.46 & 45.61 \\
\hline $6 h$ & 45.14 & 38.52 & 56.71 & 62.83 & 30.02 \\
\hline $6 \mathbf{i}$ & 78.45 & 66.90 & 95.34 & 100.00 & 56.25 \\
\hline Fluopyram & 71.37 & 62.90 & 100.00 & 100.00 & 100.00 \\
\hline
\end{tabular}

${ }^{a}$ Average of three replicates. 
表 2 对油菜菌核病原真菌有优异抑制效果的化合物的 $\mathrm{EC}_{50}$ 值

Table $2 \mathrm{EC}_{50}$ values of the compounds with excellent effects against $S$. sclerotiorum

\begin{tabular}{cc||cc}
\hline Compd. & $\begin{array}{c}\mathrm{EC}_{50}(95 \% \mathrm{CL} / \\
\left(\mathrm{mg} \cdot \mathrm{L}^{-1}\right)\end{array}$ & Compd. & $\begin{array}{c}\mathrm{EC}_{50}(95 \% \mathrm{CL}) / \\
\left(\mathrm{mg} \bullet \mathrm{L}^{-1}\right)\end{array}$ \\
\hline $\mathbf{4 a}$ & $0.305(0.214 \sim 0.393)$ & $\mathbf{5 b}$ & $0.725(0.512 \sim 0.933)$ \\
$\mathbf{4 b}$ & $0.282(0.197 \sim 0.363)$ & $\mathbf{5 c}$ & $0.671(0.468 \sim 0.868)$ \\
$\mathbf{4 d}$ & $1.063(0.757 \sim 1.367)$ & $\mathbf{5 d}$ & $0.890(0.651 \sim 1.129)$ \\
$\mathbf{4 e}$ & $0.619(0.425 \sim 0.807)$ & $\mathbf{5 f}$ & $1.166(0.844 \sim 1.491)$ \\
$\mathbf{4 f}$ & $0.808(0.586 \sim 1.027)$ & $\mathbf{5 g}$ & $0.294(0.207 \sim 0.379)$ \\
$\mathbf{4 m}$ & $0.057(0.043 \sim 0.070)$ & $\mathbf{6 c}$ & $1.420(1.064 \sim 1.792)$ \\
$\mathbf{5 a}$ & $1.385(1.042 \sim 1.742)$ & $\mathbf{6 i}$ & $0.054(0.041 \sim 0.067)$ \\
Fluopyram & $0.244(0.199 \sim 0.294)$ & &
\end{tabular}

发现相应的酰胺基在苯环间位的活性要高于对位, 但 5a 同 5d 的对比结果正好相反, 其原因值得进一步研究. 同样，如果将化合物 $\mathbf{4 a}, \mathbf{4 b}$ 的抑制活性分别同化合物 $5 \mathbf{a}, 5 \mathbf{b}$ 的抑制活性进行比较, 可知苯环间位为丙烯酰胺 基的活性高于 3-乙氧基丙酰胺基的活性.

尽管通过上述研究未能找到确定的结构与活性之 间的相互关系，但得到的一些有意义的结果可用于设计 和合成更多的类似物, 以便进一步研究其定量的结构与 活性之间的相互关系，从而发现更多的活性化合物或先 导化合物. 例如, 化合物 $\mathbf{4 m}$ 和 $\mathbf{6 i}$ 可以作为先导化合物 进行进一步的研究, 因为它们具有优异的抑菌活性和简 单的结构.

\section{3 酶的抑制活性}

为了确定琥珀酸脱氢酶是否是上述合成化合物的 靶标酶，测定了它们对琥珀酸脱氢酶的抑制活性. 以氟 吡菌酰胺为比较标准, 测定了化合物 $\mathbf{4 m}$ 和 $6 \mathbf{i}$ 对油菜菌 核病原真菌线粒体中的琥珀酸脱氢酶的抑制活性, 结果 如表 3 所示. 化合物 $4 \mathbf{m}$ 和 $6 \mathbf{i}$ 的半抑制浓度 $\left(\mathrm{IC}_{50}\right)$ 分别为 $0.202 、 0.199 \mathrm{mg} / \mathrm{L}$, 表明它们比氟吡菌酰胺 $\left(\mathrm{IC}_{50}=0.356\right.$ $\mathrm{mg} / \mathrm{L}$ )具有更好的抑制活性. 与此同时, 这些研究结果 显示, 琥珀酸脱氢酶可能是这些化合物的一个作用靶 标.

表 3 化合物 $4 \mathrm{~m}$ 和 $6 \mathbf{i}$ 对 $\mathrm{SDH}$ 的抑制活性

Table 3 Inhibitory activity of compounds $4 \mathrm{~m}$ and $\mathbf{6 i}$ against $\mathrm{SDH}$

\begin{tabular}{cc}
\hline Compd. & $\mathrm{IC}_{50}(95 \% \mathrm{CL}) /\left(\mathrm{mg} \cdot \mathrm{L}^{-1}\right)$ \\
\hline $\mathbf{4 m}$ & $0.202(0.151 \sim 0.251)$ \\
$\mathbf{6 i}$ & $0.199(0.151 \sim 0.247)$ \\
Fluopyram & $0.356(0.255 \sim 0.454)$ \\
\hline
\end{tabular}

\section{4 同源建模和分子对接分析}

为了研究琥珀酸脱氢酶是否是目标化合物潜在的 靶标酶, 对化合物 $4 \mathrm{~m}$ 和 $6 \mathbf{i}$ 的理论结合模式进行了计算 机模拟. 化合物 $\mathbf{4 m}$ 与琥珀酸脱氢酶的结合模式如图 5
所示. 化合物 $4 \mathrm{~m}$ 结合在由 SDH 的 B、C 和 D 链所组成 的活性口袋中. $4 \mathrm{~m}$ 位于一个由氨基酸残基 B/Pro-226、 B/Trp-230、B/Ile-275、C/Leu-72、C/Trp-81、C/Ile-82 所 组成的疏水性腔袋中, 形成强烈的疏水性相互作用. 详 细分析可知, $\mathbf{4 m}$ 的噻吩环可以与氨基酸残基 $\mathrm{C} / \mathrm{Arg}-88$ 形成阳离子- $\pi$ 相互作用. 更为重要的是, 化合物 $\mathbf{4 m}$ 与 $\mathrm{B} / \mathrm{Trp}-230$ 残基之间形成了一个氢键，其键长为 $0.26 \mathrm{~nm}$, 这是化合物 $\mathbf{4 m}$ 与 SDH 之间主要的相互作用. 所有这些 相互作用使得 $\mathbf{4 m}$ 与 SDH 形成了稳定的复合物. 此外, 估算的结合能是 $-31.0 \mathrm{~kJ} / \mathrm{mol}$.

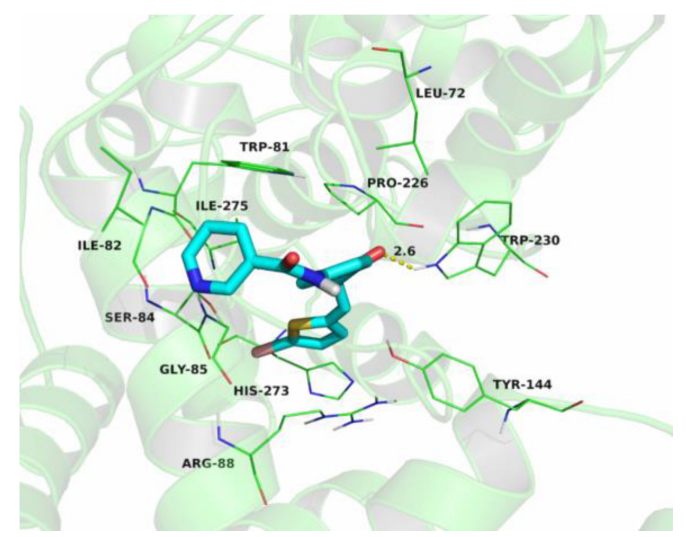

图 5 化合物 $\mathbf{4 m}$ 与 $\mathrm{SDH}$ 的理论结合模式

Figure 5 Theoretical binding mode between compound $\mathbf{4 m}$ and $\mathrm{SDH}$

化合物 6i 与琥珀酸脱氢酶的结合模式如图 6 所示. 化合物 $6 \mathbf{i}$ 结合在由 SDH 的 B、C 和 D 链所组成的活性 口袋中. $6 \mathbf{i}$ 位于一个由氨基酸残基 $\mathrm{B} / \mathrm{Pro}-226 、 \mathrm{~B} / \mathrm{Trp}-$ 230、B/Ile-275、C/Leu-72、C/Trp-81、C/Ile-82 所组成 的疏水性腔袋中, 形成强烈的疏水性相互作用. 详细分 析可知, $\mathbf{6 i}$ 的吡啶环可以与氨基酸残基 $\mathrm{C} / \mathrm{Trp}-81$ 形成 $\mathrm{CH}-\pi$ 相互作用. 更为重要的是, 在化合物 $\mathbf{6 i}$ 与 $\mathrm{B} / \mathrm{Trp}-$ 230 残基之间形成了一个氢键, 其键长为 $0.33 \mathrm{~nm}$, 这是

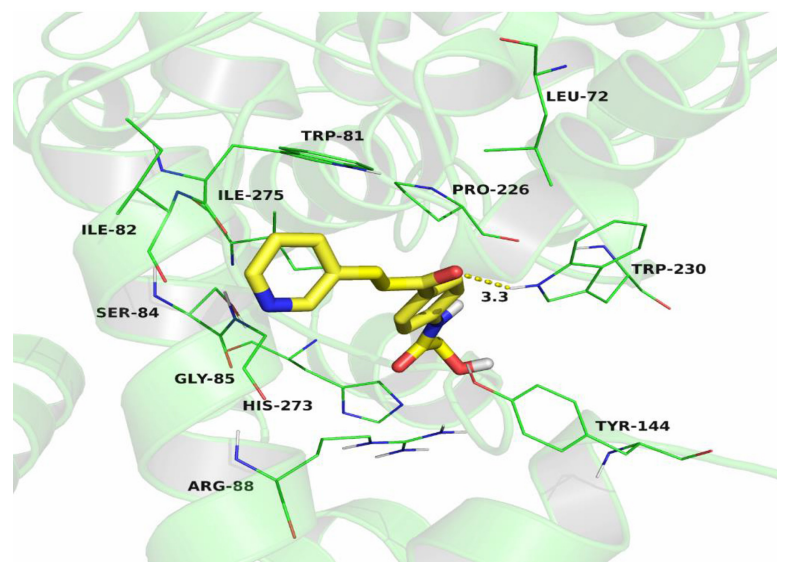

图 6 化合物 $6 \mathbf{i}$ 与 $\mathrm{SDH}$ 的理论结合模式

Figure 6 Theoretical binding mode between compound $6 \mathbf{i}$ and $\mathrm{SDH}$ 
化合物 6i 与 SDH 之间主要的相互作用. 所有这些相互 作用使得 $6 \mathbf{i}$ 与 $\mathrm{SDH}$ 形成了稳定的复合物. 此外, 估算的 结合能是 $-31.4 \mathrm{~kJ} / \mathrm{mol}$.

上述分子对接结果显示, 化合物 $\mathbf{4 m}$ 和 $6 \mathbf{i}$ 可能是 SDH 的潜在的抑制剂. 与此同时, 化合物 $\mathbf{4 m}$ 和 $\mathbf{6 i}$ 与 SDH 之间的结合能表明, 它们对 SDH 具有较强的亲和 力, 这与抑菌活性测定的实验结果相一致. 这些结果为 化合物 $4 \mathbf{m}$ 和 $6 \mathbf{i}$ 与 $\mathrm{SDH}$ 之间的相互作用给予了合理的 解释, 并为 SDH 抑制剂的研究和开发提供了一些有价 值的信息.

\section{2 结论}

设计合成了 29 个查尔酮衍生物, 并测定了它们对 5 种植物病原真菌的抑制活性. 发现了 5 个结构简单且对 油菜菌核具有优异抑制活性的化合物 $(4 \mathrm{a} 、 4 \mathrm{~b} 、 4 \mathrm{~m} 、 5 \mathrm{~g}$ 和 6i). 特别是, 化合物 $4 \mathrm{~m}$ 和 $6 \mathrm{i}$ 对油菜菌核的抑制活性 甚至超过了氟吡菌酰胺. 因此, 化合物 $\mathbf{4 m}$ 和 $6 \mathbf{i}$ 可以作 为先导化合物进一步设计和合成更多的类似物, 从而发 现更多的活性化合物. 分子对接研究结果将有助于进一 步探索上述化合物可能的杀菌机理.

\section{3 实验部分}

\section{1 仪器与试剂}

除另有说明外, 所有化学药品及溶剂均购自试剂公 司. 水稻纹枯病原真菌、小麦赤霉病原真菌、玉米小斑 病原真菌、油菜菌核病原真菌和番茄灰霉病原真菌均购 自中国农业科学院, 在 $4{ }^{\circ} \mathrm{C}$ 保存备用. 红外光谱用 Nicolet 380 红外光谱仪进行测定. ${ }^{1} \mathrm{H}$ NMR 和 ${ }^{13} \mathrm{C}$ NMR 用 AVANCE NEO 400 核磁共振仪测定, 氛代二甲亚砜 $\left(\right.$ DMSO- $\left.d_{6}\right)$ 为溶剂. 高分辨质谱用 LTQ Orbitrap Elite 质 谱仪测定. 熔点用 X-4B 显微熔点仪测定, 温度未经校 正.

\section{2 目标化合物的合成}

\subsection{1 目标化合物 $\mathbf{4 a} \sim \mathbf{4 m}$ 的合成}

将醛 $(0.01 \mathrm{~mol})$ 、氨基苯乙酮 $(0.01 \mathrm{~mol})$ 和无水乙醇 $(20 \mathrm{~mL})$ 的混合物在冰浴中搅拌, 再加入 $10 \% \mathrm{NaOH}$ 溶 液 $(5 \mathrm{~mL})$, 温度控制在 $0 \sim 5{ }^{\circ} \mathrm{C}$, 反应 $3 \sim 5 \mathrm{~h}$, 并用薄层 色谱法(TLC)监测反应进程. 反应结束后, 向反应混合 物中加入蒸馏水, 并用 $10 \%$ 的 $\mathrm{HCl}$ 溶液将混合物的 $\mathrm{pH}$ 值调至 7 左右, 有沉淀析出, 过滤并用蒸馏水洗涤得到 粗产物, 再用无水乙醇重结晶得到中间体 $\mathbf{1}$.

将中间体 $\mathbf{1}(0.005 \mathrm{~mol})$ 、二氯甲烷 $(10 \mathrm{~mL})$ 和三乙胺 $(3 \mathrm{~mL})$ 的混合物在冰浴中搅拌, 再将相应的酰氯 $(0.015$ $\mathrm{mol})$ 和二氯甲烷 $(5 \mathrm{~mL})$ 的溶液缓慢地加入到上述混合物 中, 温度控制在 $0 \sim 5{ }^{\circ} \mathrm{C}$, 反应 $2 \sim 4 \mathrm{~h}$, 并用 TLC 监测
反应进程. 反应结束后，依次用 $10 \%$ 的盐酸溶液、 $10 \%$ 的 $\mathrm{NaOH}$ 溶液和蒸馏水洗涤反应混合物至中性. 旋转蒸 发除去溶剂得到粗产物, 再用无水乙醇重结晶得到目标 化合物 $\mathbf{4 a} \sim \mathbf{4 m}$.

(E)- $N$-(3-(3-(呋喃-2-基)丙烯酰基)苯基)丙烯酰胺 (4a): 黄色粉末, 收率 $75 \%$. m.p. 166 $167{ }^{\circ} \mathrm{C} ;{ }^{1} \mathrm{H}$ NMR (400 MHz, DMSO- $\left.d_{6}\right) \delta$ : $10.42(\mathrm{~s}, 1 \mathrm{H}), 8.34(\mathrm{~s}, 1 \mathrm{H}), 8.00$ (dd, $J=8.0,1.2 \mathrm{~Hz} 1 \mathrm{H}), 7.95$ (d, $J=1.2 \mathrm{~Hz}, 1 \mathrm{H}), 7.79$ (d, $J=7.6 \mathrm{~Hz}, 1 \mathrm{H}), 7.61(\mathrm{~d}, J=15.2 \mathrm{~Hz}, 1 \mathrm{H}), 7.54$ (t, $J=8.0$ $\mathrm{Hz}, 1 \mathrm{H}), 7.48(\mathrm{~d}, J=15.2 \mathrm{~Hz}, 1 \mathrm{H}), 7.13(\mathrm{~d}, J=3.2 \mathrm{~Hz}$, $1 \mathrm{H}), 6.72(\mathrm{dd}, J=3.2,1.6 \mathrm{~Hz}, 1 \mathrm{H}), 6.46(\mathrm{dd}, J=17.2,10.0$ $\mathrm{Hz}, 1 \mathrm{H}), 6.31$ (dd, $J=17.2,2.0 \mathrm{~Hz}, 1 \mathrm{H}), 5.82$ (dd, $J=10.0$, $2.0 \mathrm{~Hz}, 1 \mathrm{H}) ;{ }^{13} \mathrm{C}$ NMR $\left(100 \mathrm{MHz}\right.$, DMSO- $\left.d_{6}\right) \delta: 188.8$, $163.9,151.5,146.8,140.0,138.5,132.1,131.1,129.9$, 127.9, 124.1, 123.9, 119.2, 118.9, 118.0, 113.7; IR (KBr) $v_{\max }: 3440,3333,1694,1659,1582,1556,1484,1385$, 964, $762,677 \mathrm{~cm}^{-1}$. HRMS (ESI) calcd for $\mathrm{C}_{16} \mathrm{H}_{14} \mathrm{NO}_{3}$ $[\mathrm{M}+\mathrm{H}]^{+}$268.0968, found 268.0966.

(E)- $N$-(3-(3-(噻吩-2-基)丙烯酰基)苯基)丙烯酰胺 (4b): 淡黄色粉末, 收率 $71 \%$. m.p. 155 157 ${ }^{\circ} \mathrm{C} ;{ }^{1} \mathrm{H}$ NMR (400 MHz, DMSO- $\left.d_{6}\right) \delta: 10.41(\mathrm{~s}, 1 \mathrm{H}), 8.29$ (t, $J=$ $2.0 \mathrm{~Hz}, 1 \mathrm{H}), 8.03(\mathrm{dd}, J=8.0,1.2 \mathrm{~Hz}, 1 \mathrm{H}), 7.95(\mathrm{~d}, J=$ $15.6 \mathrm{~Hz}, 1 \mathrm{H}), 7.82(\mathrm{t}, J=9.6 \mathrm{~Hz}, 2 \mathrm{H}), 7.71(\mathrm{~d}, J=3.6 \mathrm{~Hz}$, 1H), $7.54(\mathrm{t}, J=8.0 \mathrm{~Hz}, 1 \mathrm{H}), 7.49(\mathrm{~d}, J=15.2 \mathrm{~Hz}, 1 \mathrm{H})$, $7.22(\mathrm{dd}, J=4.8,3.6 \mathrm{~Hz}, 1 \mathrm{H}), 6.47(\mathrm{dd}, J=17.2,10.0 \mathrm{~Hz}$, $1 \mathrm{H}), 6.31$ (dd, $J=16.8,2.0 \mathrm{~Hz}, 1 \mathrm{H}), 5.81$ (dd, $J=10.0,2.0$ $\mathrm{Hz}, 1 \mathrm{H}) ;{ }^{13} \mathrm{C}$ NMR $\left(100 \mathrm{MHz}\right.$, DMSO- $\left.d_{6}\right) \delta: 188.9,163.9$, $140.1,140.0,138.5,137.5,133.6,132.2,131.0,130.0$, $129.2,127.8,124.2,124.0,120.7,119.2$; IR (KBr) $v_{\max }$ : 3434, 1682, 1659, 1608, 1587, 1535, 1478, 1409, 1384, $985,798,699 \mathrm{~cm}^{-1}$. HRMS (ESI) calcd for $\mathrm{C}_{16} \mathrm{H}_{14} \mathrm{NO}_{2} \mathrm{~S}$ $[\mathrm{M}+\mathrm{H}]^{+}$284.0740, found 284.0733.

(E)-2-((3-(3-(呋喃-2-基)丙烯酰基)苯基)氨基)-2-氧 代乙酸乙酯(4c): 浅棕色粉末, 收率 75\%. m.p. 121 $123{ }^{\circ} \mathrm{C} ;{ }^{1} \mathrm{H}$ NMR (400 MHz, DMSO- $\left.d_{6}\right) \delta: 10.35$ (s, $\left.1 \mathrm{H}\right)$, $8.26(\mathrm{~s}, 1 \mathrm{H}), 7.95(\mathrm{~d}, J=1.2 \mathrm{~Hz}, 1 \mathrm{H}), 7.87$ (dd, $J=8.0,1.2$ $\mathrm{Hz}, 1 \mathrm{H}), 7.80(\mathrm{~d}, J=8.0 \mathrm{~Hz}, 1 \mathrm{H}), 7.60(\mathrm{~d}, J=15.2 \mathrm{~Hz}$, $1 \mathrm{H}), 7.53(\mathrm{t}, J=8.0 \mathrm{~Hz}, 1 \mathrm{H}), 7.47(\mathrm{~d}, J=15.6 \mathrm{~Hz}, 1 \mathrm{H})$, $7.13(\mathrm{~d}, J=3.2 \mathrm{~Hz}, 1 \mathrm{H}), 6.72(\mathrm{dd}, J=3.2,1.6 \mathrm{~Hz}, 1 \mathrm{H})$, 4.69 (s, 2H), 2.15 (s, 3H); ${ }^{13} \mathrm{C}$ NMR (100 MHz, DMSO- $\left.d_{6}\right)$ $\delta: 188.7,170.6,166.4,151.5,146.8,139.4,138.5,131.1$, $129.9,124.1,124.0,119.2,118.8,118.0,113.7,63.0,21.0$; IR (KBr) $v_{\max }: 3448,1738,1709,1654,1600,1556,1483$, 1384, 972, 761, $728 \mathrm{~cm}^{-1}$. HRMS (ESI) calcd for $\mathrm{C}_{17} \mathrm{H}_{16^{-}}$ $\mathrm{NO}_{5}[\mathrm{M}+\mathrm{H}]^{+}$314.1023, found 314.1022. 
(E)-2-氧-2-((3-(3-(噻吩-2-基)丙烯酰基)苯基)氨基) 乙酸乙酯 (4d): 淡黄色粉末, 收率 $73 \%$. m.p. 104 $105{ }^{\circ} \mathrm{C} ;{ }^{1} \mathrm{H}$ NMR (400 MHz, DMSO- $\left.d_{6}\right) \delta: 10.34(\mathrm{~s}, 1 \mathrm{H})$, $8.22(\mathrm{t}, J=2.0 \mathrm{~Hz}, 1 \mathrm{H}), 7.95$ (d, $J=15.2 \mathrm{~Hz}, 1 \mathrm{H}), 7.90$ (dd, $J=8.0,1.2 \mathrm{~Hz}, 1 \mathrm{H}), 7.84(\mathrm{~d}, J=8.0 \mathrm{~Hz}, 1 \mathrm{H}), 7.82(\mathrm{~d}$, $J=4.8 \mathrm{~Hz}, 1 \mathrm{H}), 7.71(\mathrm{~d}, J=3.6 \mathrm{~Hz}, 1 \mathrm{H}), 7.54(\mathrm{t}, J=7.6$ Hz, 1H), 7.48 (d, $J=15.2 \mathrm{~Hz}, 1 \mathrm{H}), 7.22$ (dd, $J=4.8,3.6$ $\mathrm{Hz}, 1 \mathrm{H}), 4.69$ (s, 2H), 2.15 (s, 3H); ${ }^{13} \mathrm{C}$ NMR (100 MHz, DMSO- $\left.d_{6}\right) \delta: 188.8,170.6,166.4,140.1,139.4,138.5$, $137.5,133.7,131.0,129.9,129.3,124.2,124.2,120.6$, 119.2, 63.0, 21.0; IR (KBr) $v_{\max }: 3440,2923,2853,1746$, $1705,1658,1611,1589,1478,1384,945,804,675 \mathrm{~cm}^{-1}$. HRMS (ESI) calcd for $\mathrm{C}_{17} \mathrm{H}_{16} \mathrm{NO}_{4} \mathrm{~S}[\mathrm{M}+\mathrm{H}]^{+} 330.0795$, found 330.0790 .

(E)- $N$-(4-(3-(呋喃-2-基)丙烯酰基)苯基)丙烯酰胺 (4e): 深黄色粉末, 收率 $80 \%$. m.p. $156 \sim 158{ }^{\circ} \mathrm{C} ;{ }^{1} \mathrm{H}$ NMR (400 MHz, DMSO- $d_{6}$ ) $\delta$ : $10.52(\mathrm{~s}, 1 \mathrm{H}), 8.09$ (d, $J=$ $11.6 \mathrm{~Hz}, 2 \mathrm{H}), 7.91$ (s, 1H), 7.85 (d, $J=11.6 \mathrm{~Hz}, 2 \mathrm{H}), 7.56$ (s, 2H), 7.10 (d, $J=4.4 \mathrm{~Hz}, 1 \mathrm{H}), 6.69$ (dd, $J=4.4,2.4 \mathrm{~Hz}$, $1 \mathrm{H}), 6.48(\mathrm{dd}, J=22.4,13.2 \mathrm{~Hz}, 1 \mathrm{H}), 6.32(\mathrm{dd}, J=22.8$, $2.8 \mathrm{~Hz}, 1 \mathrm{H}), 5.82(\mathrm{dd}, J=13.2,2.4 \mathrm{~Hz}, 1 \mathrm{H}) ;{ }^{13} \mathrm{C} \mathrm{NMR}$ $\left(100 \mathrm{MHz}\right.$, DMSO- $\left.d_{6}\right) \delta: 187.4,164.0,151.6,146.3,143.7$, $132.8,131.9,130.3,130.0,128.2,119.2,119.0,117.1$, 113.4; IR (KBr) $v_{\max }: 3438,3274,1667,1599,1530,1474$, 1408, 1385, 976, $816 \mathrm{~cm}^{-1}$. HRMS (ESI) calcd for $\mathrm{C}_{16} \mathrm{H}_{14} \mathrm{NO}_{3}[\mathrm{M}+\mathrm{H}]^{+}$268.0968, found 268.0965.

(E)- $N$-(4-(3-(噻吩-2-基)丙烯酰基)苯基)丙烯酰胺 (4f): 黄色粉末, 收率 $83 \%$. m.p. $144 \sim 145{ }^{\circ} \mathrm{C} ;{ }^{1} \mathrm{H}$ NMR (400 MHz, DMSO- $\left.d_{6}\right) \delta: 10.54(\mathrm{~s}, 1 \mathrm{H}), 8.13(\mathrm{~d}, J=8.4 \mathrm{~Hz}$, 2H), $7.91(\mathrm{~d}, J=15.2 \mathrm{~Hz}, 1 \mathrm{H}), 7.86(\mathrm{~d}, J=8.8 \mathrm{~Hz}, 2 \mathrm{H})$, $7.80(\mathrm{~d}, J=4.8 \mathrm{~Hz}, 1 \mathrm{H}), 7.70(\mathrm{~d}, J=3.2 \mathrm{~Hz}, 1 \mathrm{H}), 7.59$ (d, $J=15.2 \mathrm{~Hz}, 1 \mathrm{H}), 7.21(\mathrm{dd}, J=5.2,4.0 \mathrm{~Hz}, 1 \mathrm{H}), 6.49$ (dd, $J=16.8,10.0 \mathrm{~Hz}, 1 \mathrm{H}), 6.33(\mathrm{dd}, J=16.8,1.6 \mathrm{~Hz}, 1 \mathrm{H})$, $5.84(\mathrm{dd}, J=10.0,2.0 \mathrm{~Hz}, 1 \mathrm{H}) ;{ }^{13} \mathrm{C}$ NMR $(100 \mathrm{MHz}$, DMSO- $\left.d_{6}\right) \delta: 187.5,164.1,143.9,140.3,136.7,133.2$, 132.9, 132.0, 130.8, 130.3, 129.2, 128.4, 120.7, 119.3; IR $(\mathrm{KBr}) v_{\max }: 3434,1649,1595,1526,1470,1406,1384$, $993,818 \mathrm{~cm}^{-1}$. HRMS (ESI) calcd for $\mathrm{C}_{16} \mathrm{H}_{14} \mathrm{NO}_{2} \mathrm{~S}[\mathrm{M}+$ $\mathrm{H}]^{+}$284.0740, found 284.0736.

$N$-(4-((2E,4E)-5-(呋喃-2-基)戊-2,4-二烯丙基)苯基) 丙烯酰胺 (4g): 棕色粉末, 收率 79\%. m.p. 148 150 ${ }^{\circ} \mathrm{C}$; ${ }^{1} \mathrm{H}$ NMR (400 MHz, DMSO- $d_{6}$ ) $\delta: 10.53(\mathrm{~s}, 1 \mathrm{H}), 8.05$ (d, $J=8.4 \mathrm{~Hz}, 2 \mathrm{H}), 7.86(\mathrm{~s}, 1 \mathrm{H}), 7.83(\mathrm{~d}, J=8.0 \mathrm{~Hz}, 2 \mathrm{H})$, $7.52 \sim 7.37(\mathrm{~m}, 2 \mathrm{H}), 7.09(\mathrm{~d}, J=15.6 \mathrm{~Hz}, 1 \mathrm{H}), 6.97(\mathrm{dd}$, $J=15.2,10.4 \mathrm{~Hz}, 1 \mathrm{H}), 6.74(\mathrm{~d}, J=3.2 \mathrm{~Hz}, 1 \mathrm{H}), 6.62(\mathrm{dd}$,
$J=2.8,1.6 \mathrm{~Hz}, 1 \mathrm{H}), 6.49(\mathrm{dd}, J=17.2,10.0 \mathrm{~Hz}, 1 \mathrm{H}), 6.33$ (dd, $J=16.8,1.2 \mathrm{~Hz}, 1 \mathrm{H}), 5.84$ (dd, $J=10.0,1.2 \mathrm{~Hz}, 1 \mathrm{H}$ ); ${ }^{13} \mathrm{C}$ NMR (100 MHz, DMSO- $\left.d_{6}\right) \delta: 187.8,164.1,152.5$, $145.3,143.8,143.7,133.1,132.0,130.0,128.7,128.4$, 125.6, 119.3, 113.3, 113.2; IR (KBr) $v_{\max }: 3338,1690$, $1650,1578,1549,1479,1384,999,804 \mathrm{~cm}^{-1}$. HRMS (ESI) calcd for $\mathrm{C}_{18} \mathrm{H}_{16} \mathrm{NO}_{3}[\mathrm{M}+\mathrm{H}]^{+}$294.1125, found 294.1123.

(E)- $N$-(4-(3-(吡啶-3-基)丙烯酰基)苯基)丙烯酰胺 (4h): 黄色晶体, 收率 77\%. m.p. 189 191 ${ }^{\circ} \mathrm{C} ;{ }^{1} \mathrm{H}$ NMR (400 MHz, DMSO- $\left.d_{6}\right) \delta: 10.56(\mathrm{~s}, 1 \mathrm{H}), 9.04(\mathrm{~d}, J=2.0 \mathrm{~Hz}$, $1 \mathrm{H}), 8.63(\mathrm{dd}, J=4.8,1.6 \mathrm{~Hz}, 1 \mathrm{H}), 8.37$ (dt, $J=8.0,2.0$ $\mathrm{Hz}, 1 \mathrm{H}), 8.22(\mathrm{~d}, J=8.8 \mathrm{~Hz}, 2 \mathrm{H}), 8.11(\mathrm{~d}, J=15.6 \mathrm{~Hz}$, 1H), $7.88(\mathrm{~d}, J=8.8 \mathrm{~Hz}, 2 \mathrm{H}), 7.77(\mathrm{~d}, J=15.6 \mathrm{~Hz}, 1 \mathrm{H})$, 7.51 (dd, $J=8.0,4.8 \mathrm{~Hz}, 1 \mathrm{H}), 6.49$ (dd, $J=16.8,10.0 \mathrm{~Hz}$, $1 \mathrm{H}), 6.33(\mathrm{dd}, J=16.8,2.0 \mathrm{~Hz}, 1 \mathrm{H}), 5.85(\mathrm{dd}, J=10.0,2.0$ $\mathrm{Hz}, 1 \mathrm{H}) ;{ }^{13} \mathrm{C}$ NMR $\left(100 \mathrm{MHz}\right.$, DMSO- $\left.d_{6}\right) \delta: 187.7,164.1$, $151.4,150.8,144.1,140.5,135.5,132.7,132.0,131.1$, 130.6, 128.5, 124.4, 124.3, 119.2; IR (KBr) $v_{\max }: 3434$, $3337,1691,1656,1583,1547,1484,1384,972,830 \mathrm{~cm}^{-1}$. HRMS (ESI) calcd for $\mathrm{C}_{17} \mathrm{H}_{15} \mathrm{~N}_{2} \mathrm{O}_{2}[\mathrm{M}+\mathrm{H}]^{+}$279.1128, found 279.1121 .

(E)-2-氧-2-((4-(3-(噻吩-2-基)丙烯酰基)苯基)氨基) 乙酸乙酯(4i): 黄色晶体, 收率 $78 \%$. m.p. $129 \sim 131{ }^{\circ} \mathrm{C}$; ${ }^{1} \mathrm{H}$ NMR (400 MHz, DMSO- $\left.d_{6}\right) \delta: 10.50(\mathrm{~s}, 1 \mathrm{H}), 8.12(\mathrm{~d}$, $J=8.4 \mathrm{~Hz}, 2 \mathrm{H}), 7.91$ (d, $J=15.2 \mathrm{~Hz}, 1 \mathrm{H}), 7.80$ (d, $J=5.2$ $\mathrm{Hz}, 1 \mathrm{H}), 7.77$ (d, $J=8.4 \mathrm{~Hz}, 2 \mathrm{H}), 7.70$ (d, $J=3.2 \mathrm{~Hz}, 1 \mathrm{H})$, $7.59(\mathrm{~d}, J=15.2 \mathrm{~Hz}, 1 \mathrm{H}), 7.21(\mathrm{dd}, J=5.2,3.6 \mathrm{~Hz}, 1 \mathrm{H})$, $4.71(\mathrm{~s}, 2 \mathrm{H}), 2.15(\mathrm{~s}, 3 \mathrm{H}) ;{ }^{13} \mathrm{C}$ NMR (100 MHz, DMSO- $\left.d_{6}\right)$ $\delta: 187.5,170.5,166.6,143.3,140.3,136.7,133.1,133.0$, 130.7, 130.3, 129.2, 120.7, 119.2, 63.1, 20.9; IR (KBr) $v_{\text {max }}: 3427,3331,2920,2851,1649,1605,1548,1482$, 1384, $981 \mathrm{~cm}^{-1}$. HRMS (ESI) calcd for $\mathrm{C}_{17} \mathrm{H}_{16} \mathrm{NO}_{4} \mathrm{~S}[\mathrm{M}+$ $\mathrm{H}]^{+} 330.0795$, found 330.0788 .

(E)-2-氧-2-((4-(3-(吡啶-3-基)丙烯酰基)苯基)氨基) 乙酸乙酯 $(\mathbf{4 j})$ : 黄色晶体, 收率 $70 \%$. m.p. $215 \sim 217{ }^{\circ} \mathrm{C}$; ${ }^{1} \mathrm{H}$ NMR (400 MHz, DMSO- $\left.d_{6}\right) \delta: 10.52(\mathrm{~s}, 1 \mathrm{H}), 9.04(\mathrm{~d}$, $J=2.0 \mathrm{~Hz}, 1 \mathrm{H}), 8.63(\mathrm{dd}, J=4.8,1.6 \mathrm{~Hz}, 1 \mathrm{H}), 8.37(\mathrm{dt}, J=$ $8.0,1.6 \mathrm{~Hz}, 1 \mathrm{H}), 8.21(\mathrm{~d}, J=8.8 \mathrm{~Hz}, 2 \mathrm{H}), 8.11$ (d, $J=15.6$ $\mathrm{Hz}, 1 \mathrm{H}), 7.80 \sim 7.75(\mathrm{~m}, 3 \mathrm{H}), 7.51(\mathrm{dd}, J=7.6,4.4 \mathrm{~Hz}$, $1 \mathrm{H}), 4.72(\mathrm{~s}, 2 \mathrm{H}), 2.15$ (s, 3H); ${ }^{13} \mathrm{C}$ NMR (100 MHz, DMSO- $\left.d_{6}\right) \delta: 187.8,170.6,166.7,151.4,150.8,143.5$, $140.5,135.5,132.8,131.1,130.6,124.4,124.2,119.1$, 63.0, 20.9; IR (KBr) $v_{\text {max }}: 3435,3308,2921,2852,1685$, 1651, 1597, 1542, 1406, 1384, 972, $818 \mathrm{~cm}^{-1}$. HRMS 
(ESI) calcd for $\mathrm{C}_{18} \mathrm{H}_{17} \mathrm{~N}_{2} \mathrm{O}_{4}[\mathrm{M}+\mathrm{H}]^{+} 325.1183$, found 325.1183 .

(E)- $N$-(4-(3-(呋喃-2-基)丙烯酰基)苯基)烟酰胺(4k): 黄色粉末, 收率 71\%. m.p. 182 184 ${ }^{\circ} \mathrm{C} ;{ }^{1} \mathrm{H}$ NMR (400 MHz, DMSO- $\left.d_{6}\right) \delta: 10.78(\mathrm{~s}, 1 \mathrm{H}), 9.15(\mathrm{~d}, J=2.0 \mathrm{~Hz}, 1 \mathrm{H})$, $8.80(\mathrm{dd}, J=4.8,1.6 \mathrm{~Hz}, 1 \mathrm{H}), 8.34(\mathrm{dt}, J=8.0,2.0 \mathrm{~Hz}$, $1 \mathrm{H}), 8.14(\mathrm{~d}, J=8.8 \mathrm{~Hz}, 2 \mathrm{H}), 8.00(\mathrm{~d}, J=8.8 \mathrm{~Hz}, 2 \mathrm{H}), 7.93$ (d, $J=1.6 \mathrm{~Hz}, 1 \mathrm{H}), 7.62 \sim 7.59(\mathrm{~m}, 3 \mathrm{H}), 7.12(\mathrm{~d}, J=3.2$ $\mathrm{Hz}, 1 \mathrm{H}), 6.71(\mathrm{dd}, J=3.6,2.0 \mathrm{~Hz}, 1 \mathrm{H}) ;{ }^{13} \mathrm{C}$ NMR $(100$ MHz, DMSO- $d_{6}$ ) $\delta$ : $187.5,165.0,152.8,151.7,149.3$, $146.5,143.8,136.1,133.3,130.8,130.5,130.0,124.0$, 120.2, 119.1, 117.3, 113.6; IR (KBr) $v_{\max }: 3427,1658$, 1592, 1523, 1473, 1384, 977, $831 \mathrm{~cm}^{-1}$. HRMS (ESI) calcd for $\mathrm{C}_{19} \mathrm{H}_{15} \mathrm{~N}_{2} \mathrm{O}_{3}[\mathrm{M}+\mathrm{H}]^{+}$319.1077, found 319.1075 .

(E)- $N$-(4-(3-(噻吩-2-基)丙烯酰基)苯基)烟酰胺(4I): 淡黄色粉末, 收率 74\%. m.p. 194 195 ${ }^{\circ} \mathrm{C} ;{ }^{1} \mathrm{H}$ NMR $\left(400 \mathrm{MHz}, \mathrm{DMSO}-d_{6}\right) \delta: 10.79(\mathrm{~s}, 1 \mathrm{H}), 9.15(\mathrm{~d}, J=2.0 \mathrm{~Hz}$, $1 \mathrm{H}), 8.80(\mathrm{dd}, J=4.4,1.2 \mathrm{~Hz}, 1 \mathrm{H}), 8.33(\mathrm{dt}, J=8.0,1.6 \mathrm{~Hz}$, 1H), 8.17 (d, $J=8.8 \mathrm{~Hz}, 2 \mathrm{H}), 8.00$ (d, $J=8.8 \mathrm{~Hz}, 2 \mathrm{H}), 7.93$ (d, $J=15.2 \mathrm{~Hz}, 1 \mathrm{H}), 7.80(\mathrm{~d}, J=5.2 \mathrm{~Hz}, 1 \mathrm{H}), 7.71$ (d, $J=$ $3.2 \mathrm{~Hz}, 1 \mathrm{H}), 7.63 \sim 7.59(\mathrm{~m}, 2 \mathrm{H}), 7.21$ (dd, $J=4.8,3.6 \mathrm{~Hz}$, $1 \mathrm{H}) ;{ }^{13} \mathrm{C}$ NMR (100 MHz, DMSO- $\left.d_{6}\right) \delta: 187.6,165.0$, $152.8,149.3,143.8,140.3,136.7,136.1,133.3,133.2$, 130.8, 130.7, 130.1, 129.2, 124.0, 120.7, 120.1; IR (KBr) $v_{\max }: 3337,1671,1644,1595,1524,1408,1384,973,824$ $\mathrm{cm}^{-1}$. HRMS (ESI) calcd for $\mathrm{C}_{19} \mathrm{H}_{15} \mathrm{~N}_{2} \mathrm{O}_{2} \mathrm{~S}[\mathrm{M}+\mathrm{H}]^{+}$ 335.0849 , found 335.0847 .

(E)- $N$-(4-(3-(5-澳噻吩-2-基)丙烯酰基)苯基)烟酰胺 (4m): 深黄色晶体, 收率 $65 \%$. m.p. $228 \sim 230{ }^{\circ} \mathrm{C} ;{ }^{1} \mathrm{H}$ NMR (400 MHz, DMSO- $\left.d_{6}\right) \delta: 10.79(\mathrm{~s}, 1 \mathrm{H}), 9.14$ (d, $J=$ $1.6 \mathrm{~Hz}, 1 \mathrm{H}), 8.80(\mathrm{dd}, J=4.8,1.6 \mathrm{~Hz}, 1 \mathrm{H}), 8.33$ (dt, $J=$ 7.6, $1.6 \mathrm{~Hz}, 1 \mathrm{H}), 8.17$ (d, $J=8.8 \mathrm{~Hz}, 2 \mathrm{H}), 7.99$ (d, $J=8.8$ $\mathrm{Hz}, 2 \mathrm{H}), 7.84(\mathrm{~d}, J=15.2 \mathrm{~Hz}, 1 \mathrm{H}), 7.62 \sim 7.58(\mathrm{~m}, 2 \mathrm{H})$, $7.54(\mathrm{~d}, J=4.0 \mathrm{~Hz}, 1 \mathrm{H}), 7.35(\mathrm{~d}, J=4.0 \mathrm{~Hz}, 1 \mathrm{H}) ;{ }^{13} \mathrm{C}$ NMR (100 MHz, DMSO- $\left.d_{6}\right) \delta: 187.4,165.0,152.8,149.3$, $143.9,142.2,136.1,135.7,133.8,133.1,132.5,130.7$, $130.2,124.0,121.4,120.1,116.2$; IR (KBr) $v_{\max }: 3321$, 1677, 1640, 1597, 1561, 1527, 1479, 1419, 1384, 967, 834 $\mathrm{cm}^{-1}$. HRMS (ESI) calcd for $\mathrm{C}_{19} \mathrm{H}_{14} \mathrm{BrN}_{2} \mathrm{O}_{2} \mathrm{~S}[\mathrm{M}+\mathrm{H}]^{+}$ 412.9954, found 412.9959 .

\subsection{2 目标化合物 $\mathbf{5 a} \sim \mathbf{5 g}$ 的合成}

将氨基苯乙酮 $(0.01 \mathrm{~mol})$ 、二氯甲烷 $(10 \mathrm{~mL})$ 和三乙 胺 $(3 \mathrm{~mL})$ 的混合物在冰浴中搅拌, 再将丙烯酰氯 $(0.015$ $\mathrm{mol})$ 和二氯甲烷 $(5 \mathrm{~mL})$ 的混合溶液缓慢滴加到上述混合
物中. 温度控制在 $0 \sim 5{ }^{\circ} \mathrm{C}$, 反应 $2 \sim 4 \mathrm{~h}$, 并用 TLC 监 测反应进程. 反应结束后, 依次用 $10 \%$ 的盐酸溶液、 $10 \%$ 的 $\mathrm{NaOH}$ 溶液和蒸馏水洗涤反应混合物至中性. 旋转蒸 发除去溶剂得到粗产物, 再用无水乙醇重结晶得到中间 体 2.

将中间体 $2(0.005 \mathrm{~mol})$ 溶于无水乙醇 $(20 \mathrm{~mL})$ 中, 再 加入 $10 \% \mathrm{NaOH}$ 溶液 $(5 \mathrm{~mL})$, 在摚拌条件下将相应的醛 $(0.005 \mathrm{~mol})$ 和无水乙醇 $(10 \mathrm{~mL})$ 的混合溶液缓慢加入到 上述溶液中, 温度控制在 $45 \sim 50{ }^{\circ} \mathrm{C}$, 反应 $3 \sim 5 \mathrm{~h}$, 并用 TLC 监测反应进程. 反应结束后, 向反应混合物中加入 蒸馏水, 并用 $10 \%$ 的 $\mathrm{HCl}$ 溶液将混合物的 $\mathrm{pH}$ 值调至 7 左右, 有沉淀析出, 过滤并用蒸馏水洗涤得到粗产物, 再用无水乙醇重结晶得到目标化合物 $\mathbf{5 a} \sim \mathbf{5 g}$.

(E)-3-乙氧基- $N$-(3-(3-(呋喃-2-基)丙烯酰基)苯基)丙 酰胺(5a): 浅棕色粉末, 收率 81\%. m.p. 103 105 ${ }^{\circ} \mathrm{C} ;{ }^{1} \mathrm{H}$ NMR (400 MHz, DMSO- $\left.d_{6}\right) \delta: 10.21(\mathrm{~s}, 1 \mathrm{H}), 8.29(\mathrm{~s}, 1 \mathrm{H})$, $7.94(\mathrm{~d}, J=1.2 \mathrm{~Hz}, 1 \mathrm{H}), 7.91(\mathrm{dd}, J=8.0,1.2 \mathrm{~Hz}, 1 \mathrm{H})$, $7.76(\mathrm{~d}, J=8.0 \mathrm{~Hz}, 1 \mathrm{H}), 7.60(\mathrm{~d}, J=15.6 \mathrm{~Hz}, 1 \mathrm{H}), 7.51$ (d, $J=7.6 \mathrm{~Hz}, 1 \mathrm{H}), 7.47$ (d, $J=15.2 \mathrm{~Hz}, 1 \mathrm{H}), 7.12$ (d, $J=3.2$ $\mathrm{Hz}, 1 \mathrm{H}), 6.72(\mathrm{dd}, J=3.2,1.6 \mathrm{~Hz}, 1 \mathrm{H}), 3.68(\mathrm{t}, J=6.0 \mathrm{~Hz}$, $2 \mathrm{H}), 3.45$ (q, $J=7.2 \mathrm{~Hz}, 2 \mathrm{H}), 2.58(\mathrm{t}, J=6.0 \mathrm{~Hz}, 2 \mathrm{H}), 1.10$ (t, $J=6.8 \mathrm{~Hz}, 3 \mathrm{H}) ;{ }^{13} \mathrm{C}$ NMR $\left(100 \mathrm{MHz}\right.$, DMSO- $\left.d_{6}\right) \delta$ : $188.8,170.2,151.5,146.8,140.2,138.5,131.0,129.8$, $123.8,123.5,118.9,118.8,117.9,113.7,66.4,65.9,37.7$, 15.5; IR (KBr) $v_{\text {max }}: 3434,3302,2922,2853,1667,1586$, 1552, 1487, 1407, 1384, 972, 795, $710 \mathrm{~cm}^{-1}$. HRMS (ESI) calcd for $\mathrm{C}_{18} \mathrm{H}_{20} \mathrm{NO}_{4}[\mathrm{M}+\mathrm{H}]^{+}$314.1387, found 314.1385.

(E)-3-乙氧基- $N$-(3-(3-(噻吩-2-基)丙烯酰基)苯基)丙 酰胺(5b): 黄色粉末, 收率 78\%. m.p. 86 87 ${ }^{\circ} \mathrm{C} ;{ }^{1} \mathrm{H}$ NMR $\left(400 \mathrm{MHz}\right.$, DMSO- $\left.d_{6}\right) \delta: 10.21(\mathrm{~s}, 1 \mathrm{H}), 8.24(\mathrm{~s}, 1 \mathrm{H})$, $7.96 \sim 7.93(\mathrm{~m}, 2 \mathrm{H}), 7.82 \sim 7.79(\mathrm{~m}, 2 \mathrm{H}), 7.70(\mathrm{~d}, J=3.2$ $\mathrm{Hz}, 1 \mathrm{H}), 7.52(\mathrm{~d}, J=8.0 \mathrm{~Hz}, 1 \mathrm{H}), 7.47(\mathrm{~d}, J=15.2 \mathrm{~Hz}$, $1 \mathrm{H}), 7.21(\mathrm{t}, J=4.4 \mathrm{~Hz}, 1 \mathrm{H}), 3.68(\mathrm{t}, J=6.0 \mathrm{~Hz}, 2 \mathrm{H}), 3.45$ (q, $J=6.8 \mathrm{~Hz}, 2 \mathrm{H}), 2.58$ (t, $J=6.0 \mathrm{~Hz}, 2 \mathrm{H}), 1.10$ (t, $J=6.8$ $\mathrm{Hz}, 3 \mathrm{H}) ;{ }^{13} \mathrm{C}$ NMR (100 MHz, DMSO- $\left.d_{6}\right) \delta: 188.9,170.1$, $140.2,140.1,138.5,137.4,133.6,130.9,129.8,129.3$, 123.9, 123.7, 120.7, 118.8, 66.4, 65.9, 37.7, 15.5; IR (KBr) $v_{\max }: 3435,3336,2922,1684,1662,1599,1556,1482$, $1384,756,686 \mathrm{~cm}^{-1}$. HRMS (ESI) calcd for $\mathrm{C}_{18} \mathrm{H}_{20} \mathrm{NO}_{3} \mathrm{~S}$ $[\mathrm{M}+\mathrm{H}]^{+} 330.1158$, found 330.1154 .

3-乙氧基- $N$-(3-((2E,4E)-5-(呋喃-2-基)戊-2,4-二烯丙 基)苯基)丙酰胺 $(\mathbf{5 c})$ : 棕色粉末, 收率 74\%. m.p. 80 $81{ }^{\circ} \mathrm{C} ;{ }^{1} \mathrm{H}$ NMR (400 MHz, DMSO- $d_{6}$ ) $\delta: 10.19(\mathrm{~s}, 1 \mathrm{H})$, $8.22(\mathrm{~s}, 1 \mathrm{H}), 7.87$ (dd, $J=8.0,0.8 \mathrm{~Hz}, 1 \mathrm{H}), 7.82(\mathrm{~d}, J=1.2$ $\mathrm{Hz}, 1 \mathrm{H}), 7.71(\mathrm{~d}, J=7.6 \mathrm{~Hz}, 1 \mathrm{H}), 7.56 \sim 7.47(\mathrm{~m}, 2 \mathrm{H})$, 
$7.32(\mathrm{~d}, J=14.8 \mathrm{~Hz}, 1 \mathrm{H}), 7.11(\mathrm{~d}, J=15.2 \mathrm{~Hz}, 1 \mathrm{H}), 6.98$ (dd, $J=15.6,11.2 \mathrm{~Hz}, 1 \mathrm{H}), 6.76(\mathrm{~d}, J=3.2 \mathrm{~Hz}, 1 \mathrm{H}), 6.62$ (dd, $J=3.2,1.6 \mathrm{~Hz}, 1 \mathrm{H}), 3.67(\mathrm{t}, J=6.4 \mathrm{~Hz}, 2 \mathrm{H}), 3.44$ (q, $J=6.8 \mathrm{~Hz}, 2 \mathrm{H}), 2.57(\mathrm{t}, J=6.0 \mathrm{~Hz}, 2 \mathrm{H}), 1.10(\mathrm{t}, J=6.8$ $\mathrm{Hz}, 3 \mathrm{H}) ;{ }^{13} \mathrm{C}$ NMR $\left(100 \mathrm{MHz}\right.$, DMSO- $\left.d_{6}\right) \delta: 189.3,170.1$, $152.4,145.3,144.6,140.2,138.7,129.7,129.1,125.5$, 123.7, 123.5, 118.8, 113.4, 113.2, 66.4, 65.9, 37.7, 15.5; IR (KBr) $v_{\max }: 3433,3243,2926,2865,1688,1660,1610$, 1588, 1535, 1481, 1413, 1384, 804, $706 \mathrm{~cm}^{-1}$. HRMS (ESI) calcd for $\mathrm{C}_{20} \mathrm{H}_{22} \mathrm{NO}_{4}[\mathrm{M}+\mathrm{H}]^{+} 340.1543$, found 340.1538.

(E)-3-乙氧基- $N$-(4-(3-(呋喃-2-基)丙烯酰基)苯基)丙 酰胺 (5d): 深棕色粉末, 收率 82\%. m.p. $58 \sim 60{ }^{\circ} \mathrm{C} ;{ }^{1} \mathrm{H}$ NMR (400 MHz, DMSO- $\left.d_{6}\right) \delta: 10.34(\mathrm{~s}, 1 \mathrm{H}), 8.07$ (d, $J=$ $8.8 \mathrm{~Hz}, 2 \mathrm{H}), 7.92(\mathrm{~d}, J=1.6 \mathrm{~Hz}, 1 \mathrm{H}), 7.79(\mathrm{~d}, J=8.8 \mathrm{~Hz}$, 2H), 7.56 (s, 2H), 7.10 (d, $J=2.8 \mathrm{~Hz}, 1 \mathrm{H}), 6.70$ (dd, $J=$ 3.6, $2.0 \mathrm{~Hz}, 1 \mathrm{H}), 3.68$ (t, $J=6.0 \mathrm{~Hz}, 2 \mathrm{H}), 3.44$ (q, $J=7.2$ $\mathrm{Hz}, 2 \mathrm{H}), 2.61$ (t, $J=6.4 \mathrm{~Hz}, 2 \mathrm{H}), 1.10$ (t, $J=7.2 \mathrm{~Hz}, 3 \mathrm{H})$; ${ }^{13} \mathrm{C}$ NMR (100 MHz, DMSO- $\left.d_{6}\right) \delta: 187.5,170.4,151.7$, $146.4,144.0,132.6,130.3,130.1,119.2,119.0,117.0$, $113.5,66.3,65.9,37.9,15.5$; IR (KBr) $v_{\max }: 3433,3270$, 2923, 1668, 1638, 1595, 1530, 1407, 1384, 974, $820 \mathrm{~cm}^{-1}$. HRMS (ESI) calcd for $\mathrm{C}_{18} \mathrm{H}_{20} \mathrm{NO}_{4}[\mathrm{M}+\mathrm{H}]^{+} 314.1387$, found 314.1382 .

(E)-3-乙氧基- $N$-(4-(3-(噻吩-2-基)丙烯酰基)苯基)丙 酰胺 (5e): 黄色粉末, 收率 $80 \%$. m.p. 119 $120{ }^{\circ} \mathrm{C} ;{ }^{1} \mathrm{H}$ NMR (400 MHz, DMSO- $\left.d_{6}\right) \delta: 10.34$ (s, 1H), 8.10 (d, $J=$ $8.8 \mathrm{~Hz}, 2 \mathrm{H}), 7.90(\mathrm{~d}, J=15.2 \mathrm{~Hz}, 1 \mathrm{H}), 7.80 \sim 7.78(\mathrm{~m}$, $3 \mathrm{H}), 7.69$ (d, $J=3.6 \mathrm{~Hz}, 1 \mathrm{H}), 7.58(\mathrm{~d}, J=15.2 \mathrm{~Hz}, 1 \mathrm{H})$, 7.20 (dd, $J=5.2,3.6 \mathrm{~Hz}, 1 \mathrm{H}), 3.68(\mathrm{t}, J=6.4 \mathrm{~Hz}, 2 \mathrm{H}), 3.45$ (q, $J=7.2 \mathrm{~Hz}, 2 \mathrm{H}), 2.61(\mathrm{t}, J=6.4 \mathrm{~Hz}, 2 \mathrm{H}), 1.10$ (t, $J=6.8$ $\mathrm{Hz}, 3 \mathrm{H}) ;{ }^{13} \mathrm{C}$ NMR (100 MHz, DMSO- $\left.d_{6}\right) \delta: 187.4,170.5$, $144.1,140.3,136.6,133.0,132.6,130.6,130.2,129.1$, 120.7, 118.9, 66.3, 65.9, 37.8, 15.5; IR (KBr) $v_{\max }: 3318$, 2975, 2870, 1658, 1600,1528, 1477, 1409, 1384, 972, 819 $\mathrm{cm}^{-1}$. HRMS (ESI) calcd for $\mathrm{C}_{18} \mathrm{H}_{20} \mathrm{NO}_{3} \mathrm{~S}[\mathrm{M}+\mathrm{H}]^{+}$ 330.1158 , found 330.1158 .

3-乙氧基- $N$-(4-((2E,4E)-5-(呋喃-2-基)戊-2,4-二烯丙 基)苯基)丙酰胺 (5f): 棕色粉末, 收率 81\%. m.p. 115 $116{ }^{\circ} \mathrm{C} ;{ }^{1} \mathrm{H}$ NMR (400 MHz, DMSO- $\left.d_{6}\right) \delta: 10.33(\mathrm{~s}, 1 \mathrm{H})$, $8.02(\mathrm{~d}, J=8.8 \mathrm{~Hz}, 2 \mathrm{H}), 7.81(\mathrm{~d}, J=1.6 \mathrm{~Hz}, 1 \mathrm{H}), 7.78(\mathrm{~d}$, $J=8.8 \mathrm{~Hz}, 2 \mathrm{H}), 7.50$ (dd, $J=14.8,10.8 \mathrm{~Hz}, 1 \mathrm{H}), 7.42$ (d, $J=14.8 \mathrm{~Hz}, 1 \mathrm{H}), 7.08$ (d, $J=15.6 \mathrm{~Hz}, 1 \mathrm{H}), 6.97$ (dd, $J=$ $15.2,10.4 \mathrm{~Hz}, 1 \mathrm{H}), 6.73(\mathrm{~d}, J=3.2 \mathrm{~Hz}, 1 \mathrm{H}), 6.62(\mathrm{dd}, J=$ $3.2,1.6 \mathrm{~Hz}, 1 \mathrm{H}), 3.68(\mathrm{t}, J=6.0 \mathrm{~Hz}, 2 \mathrm{H}), 3.44(\mathrm{q}, J=7.2$
$\mathrm{Hz}, 2 \mathrm{H}), 2.61$ (t, $J=6.0 \mathrm{~Hz}, 2 \mathrm{H}), 1.10(\mathrm{t}, J=6.8 \mathrm{~Hz}, 3 \mathrm{H})$; ${ }^{13} \mathrm{C}$ NMR $\left(100 \mathrm{MHz}\right.$, DMSO- $\left.d_{6}\right) \delta: 187.8,170.5,152.5$, $145.2,143.9,143.7,132.8,130.0,128.6,125.6,125.5$, $118.9,113.2,113.1,66.3,65.9,37.8,15.5$; IR (KBr) $v_{\max }$ : 3434, 3321, 2925, 1658, 1596, 1529, 1474, 1407, 1384, $998,823 \mathrm{~cm}^{-1}$. HRMS (ESI) calcd for $\mathrm{C}_{20} \mathrm{H}_{22} \mathrm{NO}_{4}[\mathrm{M}+$ $\mathrm{H}]^{+} 340.1543$, found 340.1539 .

(E)-3-乙氧基- $N$-(4-(3-(吡啶-3-基)丙烯酰基)苯基)丙 酰胺 (5g): 浅黄色粉末, 收率 73\%. m.p. $165 \sim 167{ }^{\circ} \mathrm{C} ;{ }^{1} \mathrm{H}$ NMR (400 MHz, DMSO- $\left.d_{6}\right) \delta: 10.37(\mathrm{~s}, 1 \mathrm{H}), 9.04$ (d, $J=$ $1.6 \mathrm{~Hz}, 1 \mathrm{H}), 8.62(\mathrm{dd}, J=4.8,1.6 \mathrm{~Hz}, 1 \mathrm{H}), 8.37(\mathrm{dt}, J=$ $8.0,1.6 \mathrm{~Hz}, 1 \mathrm{H}), 8.19$ (d, $J=8.8 \mathrm{~Hz}, 2 \mathrm{H}), 8.10$ (d, $J=15.6$ $\mathrm{Hz}, 1 \mathrm{H}), 7.81(\mathrm{~d}, J=8.8 \mathrm{~Hz}, 2 \mathrm{H}), 7.76(\mathrm{~d}, J=15.6 \mathrm{~Hz}$, $1 \mathrm{H}), 7.51$ (dd, $J=8.0,4.8 \mathrm{~Hz}, 1 \mathrm{H}), 3.68(\mathrm{t}, J=6.0 \mathrm{~Hz}$, $2 \mathrm{H}$ ), 3.45 (q, $J=7.2 \mathrm{~Hz}, 2 \mathrm{H}$ ), 2.62 (t, $J=6.4 \mathrm{~Hz}, 2 \mathrm{H}$ ), 1.10 (t, $J=6.8 \mathrm{~Hz}, 3 \mathrm{H}) ;{ }^{13} \mathrm{C}$ NMR $\left(100 \mathrm{MHz}\right.$, DMSO- $\left.d_{6}\right) \delta$ : $187.7,170.5,151.3,150.8,144.3,140.3,135.5,132.4$, 131.1, 130.5, 124.4, 124.3, 118.9, 66.3, 65.9, 37.8, 15.5; IR (KBr) $v_{\text {max }}: 3433,3309,2921,1697,1655,1589,1527$, 1407, 1384, 965, $820 \mathrm{~cm}^{-1}$. HRMS (ESI) calcd for $\mathrm{C}_{19} \mathrm{H}_{21} \mathrm{~N}_{2} \mathrm{O}_{3}[\mathrm{M}+\mathrm{H}]^{+}$325.1547, found 325.1541.

\section{2 .3 目标化合物 $\mathbf{6 a} \sim \mathbf{6 i}$ 的合成}

将氨基苯乙酮 $(0.01 \mathrm{~mol})$ 、二氯甲烷 $(10 \mathrm{~mL})$ 和三乙 胺 $(3 \mathrm{~mL})$ 的混合物在冰浴中搅拌, 再将 2 -氧代-2-氯乙酸 乙酯 $(0.015 \mathrm{~mol})$ 和二氯甲烷 $(5 \mathrm{~mL})$ 的混合溶液缓慢滴加 到上述混合物中. 温度控制在 $0 \sim 5{ }^{\circ} \mathrm{C}$, 反应 $3 \sim 5 \mathrm{~h}$, 并 用 TLC 监测反应进程. 反应结束后，依次用 $10 \%$ 的盐酸 溶液、 $10 \%$ 的 $\mathrm{NaOH}$ 溶液和蒸馏水洗涤反应混合物至中 性. 旋转蒸发除去溶剂得到粗产物, 再用无水乙醇重结 晶得到中间体 3.

将中间体 $3(0.005 \mathrm{~mol})$ 溶于无水乙醇 $(20 \mathrm{~mL})$ 中, 再 加入 $10 \% \mathrm{NaOH}$ 溶液 $(5 \mathrm{~mL})$, 在摚拌条件下, 将相应的 醛 $(0.005 \mathrm{~mol})$ 和无水乙醇 $(10 \mathrm{~mL})$ 的混合溶液缓慢加入 到上述溶液中, 温度控制在 $45 \sim 50{ }^{\circ} \mathrm{C}$, 反应 $4 \sim 6 \mathrm{~h}$, 并 用 TLC 监测反应进程. 反应结束后，向反应混合物中加 入蒸馏水, 并用 $10 \%$ 的 $\mathrm{HCl}$ 溶液将混合物的 $\mathrm{pH}$ 值调至 7 左右, 有沉淀析出, 过滤并用蒸馏水洗涤得到粗产物, 再用无水乙醇重结晶得到目标化合物 $\mathbf{6 a} \sim \mathbf{6 i}$.

(E)- $N$-(3-(3-呋喃-2-基)丙烯酰基)苯基)-2-羟基乙酰 胺(6a): 棕色粉末, 收率 71\%. m.p. 105 106 ${ }^{\circ} \mathrm{C} ;{ }^{1} \mathrm{H}$ NMR (400 MHz, DMSO- $\left.d_{6}\right) \delta: 9.98(\mathrm{~s}, 1 \mathrm{H}), 8.43(\mathrm{~s}, 1 \mathrm{H})$, $8.05(\mathrm{~d}, J=8.0 \mathrm{~Hz}, 1 \mathrm{H}), 7.95$ (s, $1 \mathrm{H}), 7.76(\mathrm{~d}, J=7.6 \mathrm{~Hz}$, $1 \mathrm{H}), 7.60(\mathrm{~d}, J=15.2 \mathrm{~Hz}, 1 \mathrm{H}), 7.53 \sim 7.47(\mathrm{~m}, 2 \mathrm{H}), 7.12$ (d, $J=3.2 \mathrm{~Hz}, 1 \mathrm{H}), 6.72(\mathrm{~s}, 1 \mathrm{H}), 5.75(\mathrm{t}, J=6.0 \mathrm{~Hz}, 1 \mathrm{H})$, $4.04(\mathrm{~d}, J=6.0 \mathrm{~Hz}, 2 \mathrm{H}) ;{ }^{13} \mathrm{C}$ NMR $\left(100 \mathrm{MHz}\right.$, DMSO- $\left.d_{6}\right)$ 
$\delta: 188.8,171.8,151.5,146.7,139.5,138.4,131.0,129.7$, 124.4, 123.7, 119.8, 119.0, 117.9, 113.7, 62.4; IR (KBr) $v_{\max }: 3432,3338,2928,1735,1707,1650,1579,1560$, 1487, 1384, 1055, 966, 797, $699 \mathrm{~cm}^{-1}$. HRMS (ESI) calcd for $\mathrm{C}_{15} \mathrm{H}_{14} \mathrm{NO}_{4}[\mathrm{M}+\mathrm{H}]^{+}$272.0917, found 272.0913.

(E)-2-羟基- $N$-(3-(3-(噻吩-2-基)丙烯酰基)苯基)乙酰 胺(6b): 浅绿色粉末, 收率 79\%. m.p. $167 \sim 168{ }^{\circ} \mathrm{C} ;{ }^{1} \mathrm{H}$ NMR (400 MHz, DMSO- $d_{6}$ ) $\delta: 9.95$ (s, 1H), 8.39 (s, 1H), $8.07(\mathrm{dd}, J=8.0,1.2 \mathrm{~Hz}, 1 \mathrm{H}), 7.94(\mathrm{~d}, J=15.2 \mathrm{~Hz}, 1 \mathrm{H})$, $7.82 \sim 7.79(\mathrm{~m}, 2 \mathrm{H}), 7.70(\mathrm{~d}, J=3.6 \mathrm{~Hz}, 1 \mathrm{H}), 7.53 \sim 7.47$ (m, 2H), $7.22(\mathrm{dd}, J=5.2,3.6 \mathrm{~Hz}, 1 \mathrm{H}), 5.76(\mathrm{t}, J=6.0 \mathrm{~Hz}$, $1 \mathrm{H}), 4.04(\mathrm{~d}, J=6.0 \mathrm{~Hz}, 2 \mathrm{H}) ;{ }^{13} \mathrm{C}$ NMR $(100 \mathrm{MHz}$, DMSO- $\left.d_{6}\right) \delta$ : $188.9,171.8,140.2,139.5,138.4,137.4$, $133.7,131.0,129.7,129.3,124.5,123.9,120.7,119.8$, 62.4; IR (KBr) $v_{\max }: 3423,2923,2853,1696,1652,1596$, 1532, 1407, 1384, 1039, 757, $668 \mathrm{~cm}^{-1}$. HRMS (ESI) calcd for $\mathrm{C}_{15} \mathrm{H}_{14} \mathrm{NO}_{3} \mathrm{~S}[\mathrm{M}+\mathrm{H}]^{+}$288.0689, found 288.0687.

(E)- $N$-(3-(3-(5-澳噻吩-2-基)丙烯酰基)苯基)-2-羟基 乙酰胺 $(6 \mathbf{c})$ : 浅黄色粉末, 收率 $75 \%$. m.p. $156 \sim 158{ }^{\circ} \mathrm{C}$; ${ }^{1} \mathrm{H}$ NMR (400 MHz, DMSO- $d_{6}$ ) $\delta: 9.93$ (s, $1 \mathrm{H}$ ), 8.37 (s, $1 \mathrm{H}), 8.06(\mathrm{~d}, J=7.6 \mathrm{~Hz}, 1 \mathrm{H}), 7.86(\mathrm{~d}, J=15.2 \mathrm{~Hz}, 1 \mathrm{H})$, $7.81(\mathrm{~d}, J=7.6 \mathrm{~Hz}, 1 \mathrm{H}), 7.54 \sim 7.49$ (m, 2H), 7.46 (d, $J=$ $15.2 \mathrm{~Hz}, 1 \mathrm{H}), 7.35$ (d, $J=3.6 \mathrm{~Hz}, 1 \mathrm{H}), 5.76(\mathrm{t}, J=6.0 \mathrm{~Hz}$, $1 \mathrm{H}), 4.04(\mathrm{~d}, J=6.0 \mathrm{~Hz}, 2 \mathrm{H}) ;{ }^{13} \mathrm{C}$ NMR $(100 \mathrm{MHz}$, DMSO- $\left.d_{6}\right) \delta: 188.8,171.8,142.1,139.5,138.3,136.3$, $134.2,132.6,129.7,124.6,124.0,121.4,119.8,116.4$, 62.4; IR (KBr) $v_{\max }: 3428,3347,2922,2853,1655,1586$, 1544, 1489, 1384, 1068, 968, 790, $711 \mathrm{~cm}^{-1}$. HRMS (ESI) calcd for $\mathrm{C}_{15} \mathrm{H}_{13} \mathrm{BrNO}_{3} \mathrm{~S}[\mathrm{M}+\mathrm{H}]^{+}$365.9794, found 365.9795.

$N$-(3-((2E,4E)-5-(呋喃-2-基)戊-2,4-二烯丙基)苯 基)-2-羟基乙酰胺(6d): 黄色晶体, 收率 78\%. m.p. 157 $158{ }^{\circ} \mathrm{C} ;{ }^{1} \mathrm{H}$ NMR (400 MHz, DMSO- $\left.d_{6}\right) \delta: 9.93$ (s, $1 \mathrm{H}$ ), 8.36 (s, $1 \mathrm{H}), 8.00$ (dd, $J=8.0,1.2 \mathrm{~Hz}, 1 \mathrm{H}), 7.82$ (d, $J=1.2$ $\mathrm{Hz}, 1 \mathrm{H}), 7.73(\mathrm{~d}, J=8.0 \mathrm{~Hz}, 1 \mathrm{H}), 7.56 \sim 7.48(\mathrm{~m}, 2 \mathrm{H})$, $7.33(\mathrm{~d}, J=14.8 \mathrm{~Hz}, 1 \mathrm{H}), 7.11$ (d, $J=15.2 \mathrm{~Hz}, 1 \mathrm{H}), 6.98$ (dd, $J=15.2,11.2 \mathrm{~Hz}, 1 \mathrm{H}), 6.76(\mathrm{~d}, J=3.2 \mathrm{~Hz}, 1 \mathrm{H}), 6.63$ (dd, $J=3.6,2.0 \mathrm{~Hz}, 1 \mathrm{H}), 5.74$ (t, $J=6.0 \mathrm{~Hz}, 1 \mathrm{H}), 4.03$ (d, $J=6.0 \mathrm{~Hz}, 2 \mathrm{H}) ;{ }^{13} \mathrm{C}$ NMR $\left(100 \mathrm{MHz}\right.$, DMSO- $\left.d_{6}\right) \delta: 189.3$, $171.8,152.4,145.4,144.6,139.5,138.7,129.6,129.1$, 125.6, 125.5, 124.4, 123.8, 119.7, 113.4, 113.2, 62.4; IR (KBr) $v_{\max }: 3328,2919,2852,1647,1589,1580,1551$, 1488, 1476, 1384, 1057, 1000, 754, $722 \mathrm{~cm}^{-1}$. HRMS (ESI) calcd for $\mathrm{C}_{17} \mathrm{H}_{16} \mathrm{NO}_{4}[\mathrm{M}+\mathrm{H}]^{+}$298.1074, found
298.1070.

(E)- $N$-(4-(3-呋喃-2-基)丙烯酰基)苯基)-2-羟基乙酰 胺(6e): 黄色粉末, 收率 83\%. m.p. 165 167 ${ }^{\circ} \mathrm{C} ;{ }^{1} \mathrm{H}$ NMR (400 MHz, DMSO- $\left.d_{6}\right) \delta$ : $10.08(\mathrm{~s}, 1 \mathrm{H}), 8.07(\mathrm{~d}, J=$ $8.8 \mathrm{~Hz}, 2 \mathrm{H}), 7.93$ (s, 2H), 7.90 (s, 1H), 7.57 (s, 2H), 7.11 (d, $J=3.2 \mathrm{~Hz}, 1 \mathrm{H}), 6.70(\mathrm{dd}, J=3.6,2.0 \mathrm{~Hz}, 1 \mathrm{H}), 5.73$ (t, $J=6.0 \mathrm{~Hz}, 1 \mathrm{H}), 4.05(\mathrm{~d}, J=6.0 \mathrm{~Hz}, 2 \mathrm{H}) ;{ }^{13} \mathrm{C}$ NMR $(100$ MHz, DMSO- $\left.d_{6}\right) \delta: 187.6,172.1,151.7,146.5,143.4$, 132.9, 130.4, 130.0, 119.5, 119.2, 117.1, 113.5, 62.5; IR (KBr) $v_{\max }: 3441,2922,1709,1649,1598,1541,1384$, $1029,959,820 \mathrm{~cm}^{-1}$. HRMS (ESI) calcd for $\mathrm{C}_{15} \mathrm{H}_{14} \mathrm{NO}_{4}$ $[\mathrm{M}+\mathrm{H}]^{+}$272.0917, found 272.0914.

(E)-2-差基- $\mathrm{N}$-(4-(3-(噻吩-2-基)丙烯酰基)苯基)乙酰 胺(6f): 黄色粉末, 收率 79\%. m.p. $173 \sim 174{ }^{\circ} \mathrm{C} ;{ }^{1} \mathrm{H}$ NMR $\left(400 \mathrm{MHz}\right.$, DMSO- $\left.d_{6}\right) \delta: 10.08(\mathrm{~s}, 1 \mathrm{H}), 8.10(\mathrm{~d}, J=$ $8.8 \mathrm{~Hz}, 2 \mathrm{H}), 7.93 \sim 7.89(\mathrm{~m}, 3 \mathrm{H}), 7.79(\mathrm{~d}, J=5.2 \mathrm{~Hz}, 1 \mathrm{H})$, $7.70(\mathrm{~d}, J=3.2 \mathrm{~Hz}, 1 \mathrm{H}), 7.59(\mathrm{~d}, J=15.2 \mathrm{~Hz}, 1 \mathrm{H}), 7.21$ (dd, $J=5.2,3.6 \mathrm{~Hz}, 1 \mathrm{H}), 5.74(\mathrm{t}, J=6.0 \mathrm{~Hz}, 1 \mathrm{H}), 4.06$ (d, $J=6.0 \mathrm{~Hz}, 2 \mathrm{H}) ;{ }^{13} \mathrm{C}$ NMR $\left(100 \mathrm{MHz}\right.$, DMSO- $\left.d_{6}\right) \delta: 187.5$, $172.1,143.4,140.3,136.6,133.1,132.8,130.7,130.1$, $129.2,120.7,119.5,62.5$; IR (KBr) $v_{\max }: 3434,3312,2921$, 2852, 1676, 1655, 1593, 1560, 1489, 1384, 1069, 964, 827 $\mathrm{cm}^{-1}$. HRMS (ESI) calcd for $\mathrm{C}_{15} \mathrm{H}_{14} \mathrm{NO}_{3} \mathrm{~S}[\mathrm{M}+\mathrm{H}]^{+}$ 288.0689, found 288.0688 .

(E)- $N$-(4-(3-(5-澳噻吩-2-基)丙烯酰基)苯基)-2-羟基 乙酰胺 $(6 \mathbf{6 g})$ : 棕色晶体, 收率 79\%. m.p. 181 183 ${ }^{\circ} \mathrm{C} ;{ }^{1} \mathrm{H}$ NMR (400 MHz, DMSO- $\left.d_{6}\right) \delta: 10.08(\mathrm{~s}, 1 \mathrm{H}), 8.10(\mathrm{~d}, J=$ $8.8 \mathrm{~Hz}, 2 \mathrm{H}), 7.92(\mathrm{~d}, J=8.8 \mathrm{~Hz}, 2 \mathrm{H}), 7.82(\mathrm{~d}, J=15.2 \mathrm{~Hz}$, $1 \mathrm{H}), 7.57(\mathrm{~d}, J=15.6 \mathrm{~Hz}, 1 \mathrm{H}), 7.53(\mathrm{~d}, J=4.0 \mathrm{~Hz}, 1 \mathrm{H})$, $7.34(\mathrm{~d}, J=4.0 \mathrm{~Hz}, 1 \mathrm{H}), 5.74(\mathrm{t}, J=6.0 \mathrm{~Hz}, 1 \mathrm{H}), 4.05$ (d, $J=6.0 \mathrm{~Hz}, 2 \mathrm{H}) ;{ }^{13} \mathrm{C}$ NMR $\left(100 \mathrm{MHz}\right.$, DMSO- $\left.d_{6}\right) \delta: 187.4$, $172.1,143.5,142.2,135.6,133.7,132.7,132.4,130.2$, 121.4, 119.4, 116.1, 62.5; IR (KBr) $v_{\max }: 3379,2923,1698$, $1645,1595,1530,1411,1384,1036,959,828 \mathrm{~cm}^{-1}$. HRMS (ESI) calcd for $\mathrm{C}_{15} \mathrm{H}_{13} \mathrm{BrNO}_{3} \mathrm{~S} \quad[\mathrm{M}+\mathrm{H}]^{+}$ 365.9794, found 365.9790 .

$N$-(4-((2E,4E)-5-(呋喃-2-基)戊-2,4-二烯丙基)苯 基)-2-羊基乙酰胺(6h): 棕色晶体, 收率 79\%. m.p. 163 $165{ }^{\circ} \mathrm{C} ;{ }^{1} \mathrm{H}$ NMR (400 MHz, DMSO- $\left.d_{6}\right) \delta: 10.07$ (s, $\left.1 \mathrm{H}\right)$, $8.02(\mathrm{~d}, J=8.8 \mathrm{~Hz}, 2 \mathrm{H}), 7.91(\mathrm{~d}, J=8.8 \mathrm{~Hz}, 2 \mathrm{H}), 7.81(\mathrm{~d}$, $J=1.6 \mathrm{~Hz}, 1 \mathrm{H}), 7.54 \sim 7.41(\mathrm{~m}, 2 \mathrm{H}), 7.09(\mathrm{~d}, J=15.2 \mathrm{~Hz}$, $1 \mathrm{H}), 6.97$ (dd, $J=15.6,10.4 \mathrm{~Hz}, 1 \mathrm{H}), 6.74(\mathrm{~d}, J=3.2 \mathrm{~Hz}$, $1 \mathrm{H}), 6.62(\mathrm{dd}, J=3.2,1.6 \mathrm{~Hz}, 1 \mathrm{H}), 5.74(\mathrm{t}, J=6.0 \mathrm{~Hz}$, $1 \mathrm{H}), 4.05(\mathrm{~d}, J=6.0 \mathrm{~Hz}, 2 \mathrm{H}) ;{ }^{13} \mathrm{C}$ NMR $(100 \mathrm{MHz}$, DMSO- $\left.d_{6}\right) \delta: 187.9,172.1,152.5,145.3,143.8,143.3$, 
$133.0,129.9,128.6,125.6,119.4,113.2,113.2,62.5$; IR $(\mathrm{KBr}) v_{\max }: 3310,2922,2852,1652,1580,1540,1474$, 1407, 1384, 1031, $830 \mathrm{~cm}^{-1}$. HRMS (ESI) calcd for $\mathrm{C}_{17} \mathrm{H}_{16} \mathrm{NO}_{4}[\mathrm{M}+\mathrm{H}]^{+}$298.1074, found 298.1073.

(E)-2-羟基- $N$-(4-(3-(吡啶-3-基)丙烯酰基)苯基)乙酰 胺(6i): 白色晶体, 收率 $73 \%$. m.p. 231 232 ${ }^{\circ} \mathrm{C} ;{ }^{1} \mathrm{H}$ NMR (400 MHz, DMSO- $d_{6}$ ) $\delta: 10.10$ (s, 1H), 9.04 (d, $J=$ $2.0 \mathrm{~Hz}, 1 \mathrm{H}), 8.63(\mathrm{dd}, J=4.8,1.6 \mathrm{~Hz}, 1 \mathrm{H}), 8.37$ (dt, $J=$ 8.0,2.0 Hz, 1H), 8.19 (d, $J=8.8 \mathrm{~Hz}, 2 \mathrm{H}), 8.11$ (d, $J=15.6$ $\mathrm{Hz}, 1 \mathrm{H}), 7.94$ (d, $J=8.8 \mathrm{~Hz}, 2 \mathrm{H}), 7.76$ (d, $J=16.0 \mathrm{~Hz}$, $1 \mathrm{H}), 7.51(\mathrm{dd}, J=8.0,4.8 \mathrm{~Hz}, 1 \mathrm{H}), 5.75(\mathrm{t}, J=6.0 \mathrm{~Hz}$, $1 \mathrm{H}), 4.06(\mathrm{~d}, J=6.0 \mathrm{~Hz}, 2 \mathrm{H}) ;{ }^{13} \mathrm{C} \mathrm{NMR}(100 \mathrm{MHz}$, DMSO- $\left.d_{6}\right) \delta: 187.8,172.2,151.4,150.8,143.7,140.4$, $135.5,132.7,131.1,130.4,124.4,124.3,119.4,62.5$; IR (KBr) $v_{\max }: 3381,2922,2853,1690,1659,1610,1587$, 1550, 1410, 1384, 1023, 986, $804 \mathrm{~cm}^{-1}$. HRMS (ESI) calcd for $\mathrm{C}_{16} \mathrm{H}_{15} \mathrm{~N}_{2} \mathrm{O}_{3}[\mathrm{M}+\mathrm{H}]^{+}$283.1077, found 283.1078 .

\section{3 抑菌活性的测定}

以水稻纹枯病原真菌、小麦赤霉病原真菌、玉米小 斑病原真菌、油菜菌核病原真菌和番茄灰霉病原真菌为 靶标, 采用菌丝生长速率法测定合成化合物的抑菌活 性 ${ }^{[6]}$. 将供试化合物分别溶解在 DMSO (1 mL)中, 并用 $1 \%$ Tween-80 溶液稀释至 $10 \mathrm{~mL}$, 制成浓度为 $1000 \mathrm{mg} / \mathrm{L}$ 的溶液. 将上述溶液 $(1 \mathrm{~mL})$ 加到已消毒的马铃薯葡萄糖 琼脂培养基中(PDA, $49 \mathrm{~mL}$ ). 用打孔器从培养基中切取 直径为 $5 \mathrm{~mm}$ 的菌饼, 用灭菌后的接种环将其放入 PDA 平板(直径为 $9 \mathrm{~cm}$ )中心, 在 $(28 \pm 1){ }^{\circ} \mathrm{C}$ 下培养. 作为初 步篮选, 供试化合物的浓度为 $20 \mathrm{mg} / \mathrm{L}$. 对于活性较好 的化合物, 以 $20 \mathrm{mg} / \mathrm{L}$ 为初始浓度, 采用 2 倍稀释法进 一步在 5 个不同的浓度下测定其抑制活性. 以氟吡菌酰 胺(纯度为 $99 \%$ ) 作为阳性对照, 不含供试化合物和氟吡 菌酰胺的相应溶液作为空白对照, 每处理重复 3 次. 当 空白对照的菌落直径达到 5 6 cm 时, 用十字交叉法测 量每个菌落的直径, 并用公式 $I=[(C-T) /(C-0.5)] \times$ $100 \%$ 计算校正抑制率，其中 $I$ 表示抑制率， $C$ 表示空白 对照菌落的平均直径, $T$ 表示处理菌落的平均直径, 并 用 IBM SPSS Statistics 25.0 软件计算相应的 $\mathrm{EC}_{50}$ 值.

\section{4 酶的抑制活性的测定}

\subsection{1 油菜菌核病原真菌的分离}

根据文献报道的方法 ${ }^{[36]}$, 将培养好的油菜菌核病 菌的菌丝置于研钵中, 加入液氮, 将菌丝研磨成粉状. 按照 $0.10 \mathrm{~g} / \mathrm{L}$ 的比例, 把研磨好的菌丝粉末加入到线粒 体提取缓冲溶液中 $\left(10 \mathrm{mmol} \cdot \mathrm{L}^{-1} \mathrm{KH}_{2} \mathrm{PO}_{4}, \mathrm{pH} 7.2,10\right.$ $\mathrm{mmol} \cdot \mathrm{L}^{-1} \mathrm{KCl}, 10 \mathrm{mmol} \cdot \mathrm{L}^{-1} \mathrm{MgCl}_{2}, 0.5 \mathrm{~mol} \cdot \mathrm{L}^{-1}$ 蔗糖, $0.2 \mathrm{mmol} \cdot \mathrm{L}^{-1} \mathrm{EDTA}, 2 \mathrm{mmol} \cdot \mathrm{L}^{-1}$ 苯甲磺酰氟). 在 $4{ }^{\circ} \mathrm{C}$ 、 $8500 \mathrm{r} / \mathrm{min}$ 转速下, 离心 $10 \mathrm{~min}$, 重复 2 次, 得到澄清的 线粒体提取液. 弃去离心管中的残渣, 在 $4{ }^{\circ} \mathrm{C}$ 、 10000 $\mathrm{g}$ 转速下, 继续离心线粒体提取液 $20 \mathrm{~min}$ 后, 线粒体聚 集在离心管的底部. 弃去上层清液, 将底部的线粒体用 相同的缓冲液配成 $10 \mathrm{mg} / \mathrm{mL}$ 的悬浮液, 在 $-80{ }^{\circ} \mathrm{C}$ 下保 存备用.

\subsection{2 琥珀酸一泛醌一二氯酚靛酚活性抑制测定}

将待测样品溶解于 DMSO 中, 采用 2 倍稀释法配成 6 个不同浓度的待测溶液，其浓度范围为 $4.4 \sim 150$ $\mu \mathrm{mol} \cdot \mathrm{L}^{-1}$, 以 $\mathrm{DMSO}$ 为空白对照. 用上述提取线粒体的 缓冲液将备用的线粒体悬浮液稀释 20 倍, 加入 10 $\mathrm{mmol} \cdot \mathrm{L}^{-1}$ 琥珀酸, 在 $30{ }^{\circ} \mathrm{C}$ 下预激活 $30 \mathrm{~min}$. 同时, 将 96 孔板在 $30{ }^{\circ} \mathrm{C}$ 下预热 $10 \mathrm{~min}$. 将 $10 \mu \mathrm{L}$ 已经预激活的 线粒体悬浮液加入到 $200 \mu \mathrm{L}$ 用于酶活测定的缓冲液中 $\left(50 \mathrm{mmol} \cdot \mathrm{L}^{-1}\right.$ 磷酸钠, $\mathrm{pH} 7.2,250 \mathrm{mmol} \cdot \mathrm{L}^{-1}$ 蔗糖, 3 $\mathrm{mmol} \cdot \mathrm{L}^{-1} \mathrm{NaN}_{3}, 10 \mathrm{mmol} \cdot \mathrm{L}^{-1}$ 琥珀酸), 补充 $140 \mu \mathrm{mol} \cdot$ $\mathrm{L}^{-1}$ 二氯酚靛酚(DCPIP)和 $1 \mathrm{mmol} \cdot \mathrm{L}^{-1} 2,3$-二甲氧基-5甲基-1,4-苯醌 $\left(\mathrm{Q}_{0}\right)$, 再加入 $2 \mu \mathrm{L}$ 待测样品溶液, 在 $30{ }^{\circ} \mathrm{C} 、 595 \mathrm{~nm}$ 处测定溶液的吸光度值. 根据测得的 OD 值, 用 IBM SPSS Statistics 25.0 软件计算待测化合物的 $\mathrm{IC}_{50}$ 值.

\section{5 同源性建模和分子对接}

\subsection{1 同源性建模}

通过 NCBI 蛋白质数据库(http://www.ncbi.nlm.nih. gov/protein/)找到油菜菌核病原真菌中琥珀酸脱氢酶的 氨基酸序列. 本文采用 Birren 报道的 XP_001591238、 XP_001594577、XP_001597467 和 XP_001593251 序列. 以猪心脏的 SDH (PDB ID:1ZOY) 作为模板, 采用 SWISS-MODEL 全自动蛋白质结构同源性建模服务器 (http://swissmodel.expasy.org/)构建油菜菌核病菌 SDH 的三维结构.

\section{5 .2 分子对接}

为了研究化合物 $4 \mathrm{~m}$ 和 $6 \mathbf{i}$ 与 $\mathrm{SDH}$ 的结合模式, 采 用 Autodock vina 1.1.2 软件对其进行分子对接研究. 采 用 ChemBioDraw Ultra 14.0 画出化合物的结构, 然后用 ChemBio3D Ultra 14.0 转化为三维结构, 并使用 MMFF94 力场进行优化. 采用 Autodock Tools 1.5.6 程序 包生成对接输入文件. SDH 活性位点坐标设置为: center_x $=86.459$, center_y $=65.6$, center_z $=85.537$; 尺寸 为 size $\_x=15$, size $\_y=15$, size $z=15$. 为了提高计算的 准确度, 将参数 exhaustiveness 设置为 20. 使用 Vina 对 接, 其他参数均采用默认值. 选取打分值最高的构象用 
PyMoL 1.7.6 软件(http://www.pymol.org/)进行结果分 析.

\section{辅助材料(Supporting Information) 所有目标化合物} 的 IR、 ${ }^{1} \mathrm{H}$ NMR、 ${ }^{13} \mathrm{C}$ NMR 和 HRMS 图谱. 这些材料可 以免费从本刊网站(http : //sioc-journal. $\mathrm{Cn} /$ )上下载.

\section{References}

[1] Chen, Y.; Zhang, A. F.; Wang, W. X.; Zhang, Y.; Gao, T. C. Ann. Appl. Biol. 2012, 161, 247.

[2] Yanase, Y.; Kishi, J.; Inami, S.; Katsuta, H.; Yoshikawa, Y. J. Pestic. Sci. 2013, 38(4), 188.

[3] Veloukas, T.; Karaoglanidis, G. S. Pest Manage. Sci. 2012, 68(6), 858.

[4] Xiong, L.; Shen, Y. Q., Jiang, L. N.; Zhu, X. L.; Yang, W. C.; Huang, W.; Yang, G. F. ACS Symp. Ser. 2015, 1204, 175.

[5] Xiong, L.; Li, H.; Jiang, L. N.; Ge, J. M.; Yang, W. C.; Zhu, X. L.; Yang, G. F. J. Agric. Food. Chem. 2017, 65, 1021.

[6] Jin, H.; Zhou, J. Y.; Pu, T.; Zhang, A. G.; Gao, X. H.; Tao, K.; Hou, T. P. Bioorg. Chem. 2017, 73, 76.

[7] Yao, T. T.; Xiao, D. X.; Li, Z. S.; Cheng, J. L.; Fang, S. W.; Du, Y. J.; Zhao, J. H.; Dong, X. W.; Zhu, G. N. J. Agric. Food Chem. 2017, $65,5397$.

[8] Yao, T. T.; Fang, S. W.; Li, Z. S.; Xiao, D. X.; Cheng, J. L.; Ying, H. Z.; Du, Y. J.; Zhao, J. H.; Dong, X. W. J. Agric. Food Chem. $\mathbf{2 0 1 7}, 65,3204$

[9] Zhang, X. X.; Jin, H.; Deng, Y. J.; Gao, X. H.; Li, Y.; Zhao, Y. T.; Tao, K.; Hou, T. P. Chin. Chem. Lett. 2017, 28, 1731.

[10] Wang, H. Y.; Gao, X. H.; Zhang, X. X.; Jin, H.; Tao, K.; Hou, T. P. Med. Chem. Lett. 2017, 27, 90.

[11] Zhang, A. G.; Zhou, J. Y.; Tao, K.; Hou, T. P.; Jin, H. Bioorg. Med. Chem. Lett. 2018, 28, 3042.

[12] Zhang, A. G.; Yue, Y.; Yang, Y. H.; Yang, J.; Tao, K.; Jin, H.; Hou, T. P. Pestic. Biochem. Phys. 2019, 158, 175.

[13] Sivakumar, P. M.; Ganesan, S.; Veluchamy, P.; Doble, M. Chem. Biol. Drug Des. 2010, 76, 407.

[14] Bandgar, B. P.; Patil, S. A.; Korbad, B. L.; Nile, S. H.; Khobragade, C. N. Eur. J. Med. Chem. 2010, 45, 2629.

[15] Rane, R. A.; Telvekar, V. N. Bioorg. Med. Chem. Lett. 2010, 20, 5681.

[16] Solankee, A.; Kapadia, K.; Ćirić, A.; Soković, M.; Doytchinova, I.; Geronikaki, A. Eur. J. Med. Chem. 2010, 45, 510.

[17] De Carvalho Tavares, L.; Johann, S.; De Almeida Alves, T. M.; Guerra, J. C.; De Souza-Fagundes, E. M.; Cisalpino, P. S.; Bortoluzzi, A. J.; Caramori, G. F.; De Mattos Piccoli, R.; Braibante, H. T. S.; Braibante, M. E. F.; Pizzolatti, M. G. Eur. J. Med. Chem. 2011, 46, 4448 .
[18] Sugamoto, K.; Matsusita, Y.; Matsui, K.; Kurogi, C.; Matsui, T. Tetrahedron 2011, 67, 5346.

[19] Chu, W. C.; Bai, P. Y.; Yang, Z. Q.; Cui, D. Y.; Hua, Y. G.; Yang, Y.; Yang, Q. Q.; Zhang, E.; Qin, S. S. Eur. J. Med. Chem. 2018, 143, 905 .

[20] Zhou, D. G.; Xie, D. D.; He, F. C.; Song, B. A.; Hu, D. Y. Bioorg. Med. Chem. Lett. 2018, 28, 2091.

[21] Tomar, V.; Bhattacharjee, G.; Kamaluddin; Rajakumar, S.; Srivastava, K.; Puri, S. K. Eur. J. Med. Chem. 2010, 45, 745.

[22] Wanare, G.; Aher, R.; Kawathekar, N.; Ranjan, R.; Kaushik, N. K.; Sahal, D. Bioorg. Med. Chem. Lett. 2010, 20, 4675.

[23] Nazarian, Z.; Emami, S.; Heydari, S.; Ardestani, S. K.; Nakhjiri, M.; Poorrajab, F.; Shafiee, A.; Foroumadi, A. Eur. J. Med. Chem. 2010, 45, 1424.

[24] Sharath, V.; Kumar, H. V.; Naik, N. J. Pharm. Res. 2013, 6, 785.

[25] El Sayed Aly, M. R.; El Razek Fodah, H. H. A.; Saleh, S. Y. Eur. J. Med. Chem. 2014, 76, 517.

[26] Fu, Y.; Sheng, L.; Gao, L.; Li, J.; Sun, L. Chin. J. Org. Chem. 2019, 39, 1029 (in Chinese) (付洋，盛丽，高立信，李佳，孙良鹏，有机化学, 2019, 39, 1029.)

[27] Pontes, O.; Costa, M.; Santos, F.; Sampaio-Marques, B.; Dias, T.; Ludovico, P.; Baltazar, F.; Proença, F. Eur. J. Med. Chem. 2018, 157, 101.

[28] Sheng, Q.; Zhao, W. Q.; Zeng, M.; Xie, Z. P.; Xia, Y. P.; Cui, D. M. Chin. J. Org. Chem. 2019, 39, 703 (in Chinese). (盛琦威, 赵婉秋, 曾明, 谢中袍, 夏雅平, 崔冬梅, 有机化学, 2019, 39(3), 703.)

[29] Bano, S.; Javed, K.; Ahmad, S.; Rathish, I. G.; Singh, S.; Chaitanya, M.; Arunasree, K. M.; Alam, M. S. Eur. J. Med. Chem. 2013, 65, 51.

[30] Lin, Y. M.; Zhou, Y.; Flavin, M. T.; Zhou, L. M.; Nie, W.; Chen, F. C. Bioorg. Med. Chem. 2002, 10, 2795.

[31] Ono, M.; Hori, M.; Haratake, M.; Tomiyama, T.; Mori, H.; Nakayama, M. Bioorg. Med. Chem. 2007, 15, 6388.

[32] Dos Santos, M. B.; Bertholin Anselmo, D.; De Oliveira, J. G.; Jardim-Perassi, B. V.; Alves Monteiro, D.; Silva, G.; Gomes, E.; Lucia Fachin, A.; Marins, M.; De Campos Zuccari, D. A. P.; Octavio Regasini, L. J. Enzyme Inhib. Med. Chem. 2019, 34, 1093.

[33] Karaman, I.; Gezegen, H.; Gürdere, M. B.; Dingil, A.; Ceylan, M. Chem. Biodiversity 2010, 7, 400.

[34] Zhu, M.; Wang, J.; Xie, J. B.; Chen, L. P.; Wei, X. Y.; Jiang, X.; Bao, M.; Qiu, Y. Y.; Chen, Q.; Li, W. L.; Jiang, C. X.; Zhou, X. O.; Jiang, L. P.; Qiu, P. H.; Wu, J. Z. Eur. J. Med. Chem. 2018, 157, 1395.

[35] Chu, W. C.; Bai, P. Y.; Yang, Z. Q.; Cui, D. Y.; Hua, Y. G.; Yang, Y.; Yang, Q, Q.; Zhang, E.; Qin, S. S. Eur. J. Med. Chem. 2017, 143, 905.

[36] Ye, Y. H.; Ma, L.; Dai, Z. C.; Xiao, Y.; Zhang, Y. Y.; Li, D. D.; Wang, J. X.; Zhu, H. L. J. Agric. Food Chem. 2014, 62, 4063. 